\title{
3-D Inlet Shock-Boundary Layer Interactions - PIV Database for the Second SBLI Workshop
}

\author{
W. Ethan Eagle*, James F. Driscoll ${ }^{\dagger}$, \\ University of Michigan, Ann Arbor MI 48109, \\ John A. Benek \\ U.S. Air Force Research Laboratory, Wright-Patterson Air Force Base, OH, 45433
}

\begin{abstract}
A unique data base of stereo-PIV velocity measurements has been recorded and analyzed in order to understand the complex flow separation patterns that occur due to a threedimensional shock-boundary layer interaction (SBLI) within a rectangular inlet. The database will be used for the Second Workshop of CFD predictions of SBLI. This dataset provides the first complete measurements of a three-dimensional SBLI - that is, all three velocity components were measured in all three orthogonal planes (a total of 16 data planes total). An experimental methodology was followed such that the data collected would be of maximum utility for CFD comparisons. These metrics include velocity contours that define the free stream core, the characteristic sonic line and the sizes of corner vortices. The purpose of this paper is to provide methodology and results from the boundary conditions and geometry of the experiment; a later paper will provide the full experimental findings. Access to a website containing the CAD drawings of the experiment, calculations of the metrics of interest, and other information for potential participants in the workshop is available at https://ctools.umich.edu/portal. Access can be granted by contacting the authors at 3d.sbli.workshop@umich.edu.
\end{abstract}

\section{A.Introduction}

The Air Force is interested in high-speed vehicles capable of striking targets with high precision anywhere in the world and in a fleet of reliable, reusable vehicles for space access. The Air Force Research Laboratory (AFRL) is developing air-breathing concept systems to meet these needs. A critical component of this development is the ability to accurately predict the performance of the propulsion flow paths, especially, the inlet system flow stability, which is determined by internal shock systems. Critical physics must be accurately simulated by a high fidelity, physics-based design capability including the interaction between shocks with the inlet wall, boundary layers, and corner flows. The fidelity of the simulation can only be assessed by comparison with validation quality data from experiments that measure all of these key physical phenomena. Such data does not, in general, exist. Therefore, the AFRL in collaboration with the University of Michigan has undertaken to obtain such data [1-3].

This paper provides details for this first set of validation data, which was obtained for an incident oblique shock impinging on a turbulent boundary layer in a three-dimensional rectangular inlet configuration. Contrary to many previous studies that isolated the SBLI 'of interest' from three-dimensional influence, this flow intentionally constitutes a composite of unit problems (a 'unit' problem is one where there is a

\footnotetext{
* Graduate Research Assistant, Aerospace Engineering Department, 1320 Beal Avenue \#2004 FXB Ann Arbor, Michigan 48105, AIAA Student Member

${ }^{\dagger}$ Professor, Aerospace Engineering Department, AIAA Fellow

$\$$ Director, Computational Sciences Center, AFRL Air Vehicles Directorate, AIAA Fellow.
} 
single, frequently small, region of interest). The test apparatus incorporates many critical features of real non-axisymmetric high speed inlets including shock boundary layer interactions (SBLI) on the side walls (Swept-Shock and/or Fin-Plate), the bottom wall(an Oblique Impinging Shock), and in the corner formed by the interaction of the bottom and side walls, as well as other geometries[4-14]. To the authors' knowledge (see section B.4), this is the first such dataset of it's kind. Furthermore, care has been taken to rigorously document variability and repeatability, making this dataset an excellent candidate not only for phenomenological description, but also computational validation.

To continue the assessment of the state-of-the-art and state-of-the-practice approaches, the second workshop on computational fluid dynamics (CFD) prediction of shock boundary layer interactions (SBLIs) will be held at the 2013 AIAA Fluid Dynamics meeting in San Diego which is planned to run from June $24-27^{\text {th }}$. The purpose of the workshop is to share prediction methodologies, assess the state of the art in SBLI prediction and determine the most promising methods. One motivation for the second workshop is a lesson that was learned from the first workshop and was reported by Benek [1] to be: "The extent of the coupling between SBLI in the corner flow and tunnel center was not fully appreciated at the time of the measurements. As a result of this lack of understanding, critical corner flow data was not available. Without such information, turbulence models for this type of flow cannot be properly assessed."

That scarce attention has been paid to the effect of the sidewalls in an enclosed inlet on SBLI structure is not surprising. Simulations of traditional SBLI phenomenon often assume two dimensional flow, and often experiments occur in large aspect ratio wind tunnels where the area of interest is confined to the central third (or less) of the span. However, a two dimensional understanding of the challenges of 'unit physics' type problems is not readily translatable to real systems. Interest in validated CFD for such full three-dimensional inlets is ever increasing. The continuing lack of validation-quality experiments using simple geometries (e.g. full span, and without 'aerodynamic fences' ) is our primary concern.

The present study is a logical extension of the first workshop on CFD prediction of SBLI that was held at the 48th AIAA Aerospace Sciences Meeting in January of 2010. Several CFD solutions were submitted which corresponded to four experimentally measured SBLIs. Prior to the meeting four test cases were identified and contributions of solutions were solicited from the aerospace community. A total of 36 solutions from 11 contributors and 9 organizations were submitted. Two summary papers by Benek [1] and DeBonis et al. [2] described the general conclusions. An assessment of the submitted solutions first was performed. This consisted of an assessment of the experimental data, the development of an error metric and the assessment of the CFD data using the error metric. The experimental data for the four test cases came from two different sources: the Institut Universitaire des Systemes Thermiques Industriels (IUSTI) in Marseilles, France and the University of Michigan (UMich)[3]. The data used for comparison consisted of velocities and turbulent stresses measured on a plane parallel with the free stream flow and the tunnel sidewall. An uncertainty analysis of the experimental data had been previously conducted by each of the research groups involved. As part of this work a statistical uncertainty analysis of the UMich. data was performed.

DeBonis et al. [2] concluded that "CFD solutions provided very similar levels of error and in general it was difficult to discern clear trends in the data. For the Reynolds Averaged Navier-Stokes methods the choice of turbulence model appeared to be the largest factor in solution accuracy. Large-eddy simulation methods produced error levels similar to RANS methods but provided superior predictions of normal stresses". In Ref. [1], Benek concluded that the major lessons learned were:

1. High quality, incident SBLI experimental data has been obtained at Michigan and Marseilles that focus on the SBLI interaction region in the center of the tunnel. The extent of the coupling between the corner flow and SBLI near the tunnel center was not fully appreciated at the time of the 
measurements. As a result of this lack of understanding, critical corner flow data is not available. Without such information, turbulence models for this type of flow cannot be properly assessed.

2. Detailed and comprehensive boundary condition data is as important as measurements in the regions of important physics.

3. Consistency checking of critical measurements is very important. Independent measurement methods are highly recommended.

4. Quantitative measurements such as Schlieren, oil flow, pressure sensitive paint, etc. should not be neglected.

5. RANS and LES models were used that were three-dimensional (with one exception) and completely viscous. The simulations showed that with current resolution, there is a significant variation in the turbulence models predictions in the interaction region.

6. Grid adaptation gave indications of improving agreement with the experiment in the interaction region by concentrating points in regions with larger numerical error. This result suggests that grid effects are at least as important as the turbulence models effects.

7. While the LES and RANS/LES hybrid simulations presented at the workshop cannot be judged to be better that the RANS simulations, the RANS/LES hybrid methods shows promise for complex flows.

8. It is recommended that in the design of the experiments, a computational group should be part of the team.

9. Measurement and experimental error estimates must be provided as part of the data set.

The workshop demonstrated that the requirement for validation quality experiments surpasses those of high quality phenomenological experiments. This difference consists primarily of detailed and comprehensive boundary condition measurements as well as a healthily skeptical attitude towards all experimental observations with regard to precision and uncertainty. In addition, it was determined that qualitative measurements such as schlieren and oil flow should also be included as an integral part of the validation data set. Measurements for the first workshop focused on obtaining data near the tunnel stream-wise symmetry plane. Since the flow was symmetric there, it was assumed that the flow was essentially two dimensional. However, it was found that the flow was significantly influenced by separations caused by the shock boundary layer interaction with the corner flows at the side and bottom wall interactions. This interaction was not characterized by the first measurements in sufficient detail. A partial consequence of this behavior is that the comparisons of simulations and data were not as informative as had been anticipated. For example, the LES or hybrid LES models could not be judged superior to the RANS predictions. It was determined that a second set of experimental data focused on obtaining more complete characterization of the flow, including accurate measurements of the boundary conditions at multiple locations, would be required to make a more refined assessment of CFD simulation capacity for the unit problem.

This paper describes the experimental Stereo-PIV database that recently was recorded at the University of Michigan to specifically consider a 3-D SBLI in a rectangular inlet. Of particular interest is the interaction between the bottom wall separation region, the sidewall separation region and the effects of corner flows. The geometry and experimental test procedures were selected specifically to address the above-stated lessons from the first workshop. The Michigan database consists of two sections. Section One lists the inflow and outflow boundary conditions, as well as the exact geometry of the experiment. It describes the measurement grid used to acquire PIV data and the uncertainty in the measurements. A standard grid of the computational domain in a coarse and fine resolution will also be available on the website. The present paper reports a subset of what is included in Section One, but it does not attempt to report all of the boundary conditions, or details of the computational grid. The reader must access Section One to download all of the information. Section Two of the database contains the stereo-PIV measurements of all three velocity components that were made on 16 different planes. In particular, 7 of these planes represent the first data set, to the authors' knowledge, to record PIV measurements in a field 
of view inclusive of the centerline and sidewall through an SBLI forming a 'corner interaction' exhibiting strongly three-dimensional character. Section Two also includes wall pressure data, and oil flow images of the skin friction lines on the bottom wall and sidewall. However, data from Section Two will not be made available until a date to be set by the workshop organizers.

\section{B. Experimental Facilities}

The first set of stereo-PIV measurement made in the Michigan Glass Wind Tunnel were obtained on the center line of the nominal Mach 2.75 wind tunnel, and they were reported at the first SBLI-workshop [1, 2]. It was understood that away from the centerline the rectangular test section contains a 3-D SBLI because the incident and reflected shocks interacted with the walls and corner flows. Therefore this 3-D SBLI problem was chosen for detailed study.

\section{B1. Michigan Glass Wind Tunnel}

The facility is a vacuum-driven supersonic tunnel with a Mach 2.75 nozzle shown in Fig. 1. The run time is up to ten minutes. The stagnation temperature was the measured room temperature of $295.7 \mathrm{~K}$ and the stagnation pressure is the measured room pressure of $98.0 \mathrm{kPa}$. An incident oblique shock is generated by the six degree wedge which is mounted under the upper wall on a strut, as seen in Fig. 1. It is suspended from the top wall of the tunnel by a strut. This offset helps to remove the fluctuating influence of the developing top wall boundary layer. The top face of the wedge is angled at 2 degrees to provide an expansion that helps to prevent the flow from choking in this narrow channel due to blockage.

The nozzle block is a two-dimensional converging diverging nozzle designed using the NOZCX Fortran code using the method of characteristics. The nozzle over-expands slightly to accommodate for boundary layers. The geometry of the converging-diverging nozzle is also potentially critical for computational simulation of the present geometry and an under-sampled array of 163 defining coordinates is available online. The total length of the nozzle is $615 \mathrm{~mm}$. From the nozzle exit, the boundary layer continues to develop in the rectangular test section for a length of $172 \mathrm{~mm}$ before meeting with the suspended wedge at the start of the 'test section.'
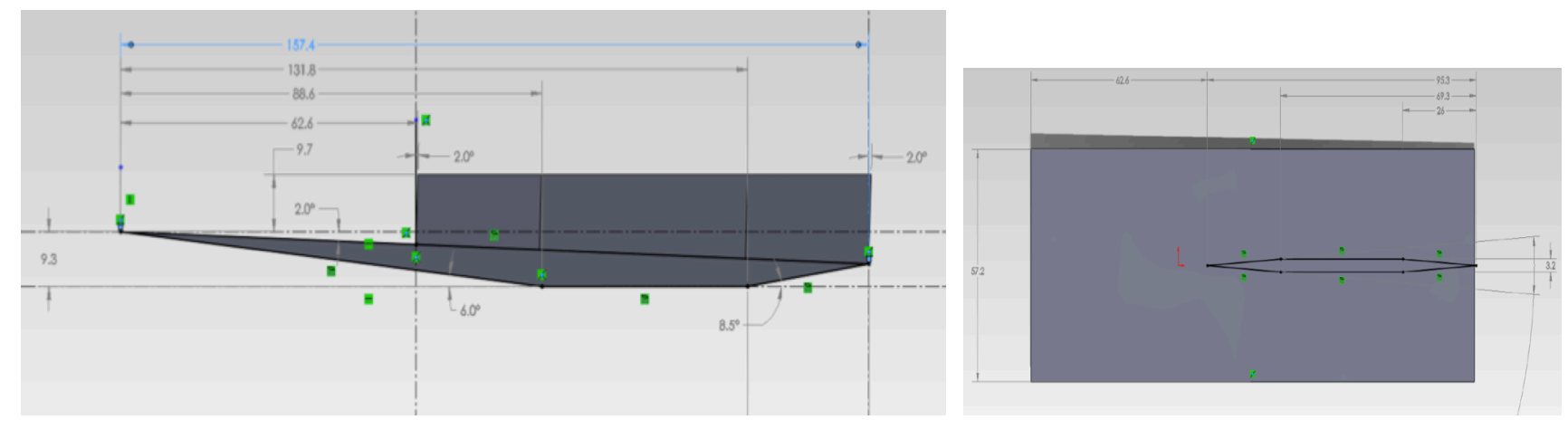

Figure 1. Dimensions of the shock wave generator (wedge and strut) that is mounted on the upper wall.

A slight deviation in the height of the tunnel test section was recently measured 'as installed' compared to as designed. The test section, displayed schematically in Fig 2a, as measured, is 2.25 inches wide by 2.72 inches high $(57.15 \times 69.08 \mathrm{~mm})$. Three upstream inflow boundary planes are shown in red in Figure $2 \mathrm{a}$. They are located at $-144 \mathrm{~mm}-72 \mathrm{~mm}$ and $0 \mathrm{~mm}$ and contain information about the core-flow and bottom and sidewall boundary layers. Figure $2 b$ indicates the $x$-location of the 13 PIV data plane measurements 
which will be made available in Part II in relation to the six-degree wedge used as a shock generator, and the location of the inviscid shock predicted from simple 2D theory.

The working fluid consists of conditioned air from the laboratory. Flow straightening devices (hexagonal meshes) are used upstream of the nozzle to assure flow uniformity. The unit Reynolds number for the experiment was $8.9 \times 10^{6} / \mathrm{m}$. Optical access is provided by glass side walls along the entire length of the test section and by an acrylic panel located in the floor of the tunnel along the centerline from $\mathrm{x}: 45 \mathrm{~mm}$ to $140 \mathrm{~mm}$ by z: $12 \mathrm{~mm}$ to $43 \mathrm{~mm}$.

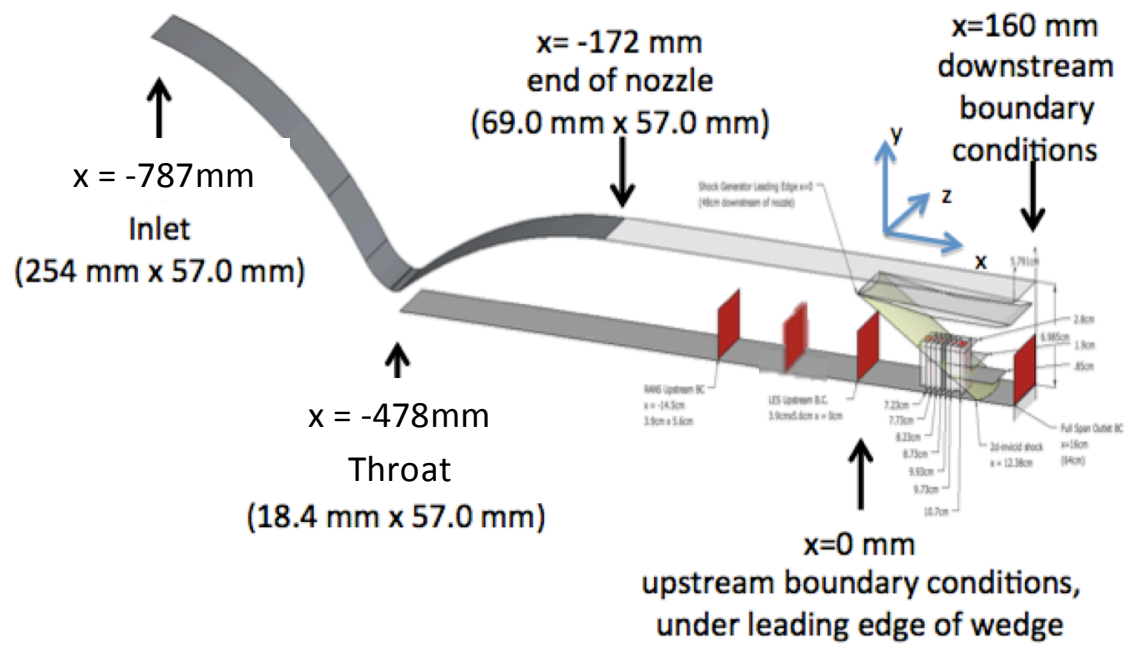

Figure 2a. Schematic of the Michigan Glass Wind Tunnel with flow from left to right. Also shown is the left handed coordinate system was chosen based on the PIV data to keep velocities positive always away from the wall; [x,y,z] corresponds to the the streamwise coordinate $\mathrm{x}$, vertical coordinate $\mathrm{y}$ and tunnel span coordinate $\mathrm{z}$. Note three upstream inflow boundaries at $-144,-72$ and $0 \mathrm{~mm}$ from the wedge leading edge. Zoom view of interaction in Fig $1 \mathrm{~b}$.

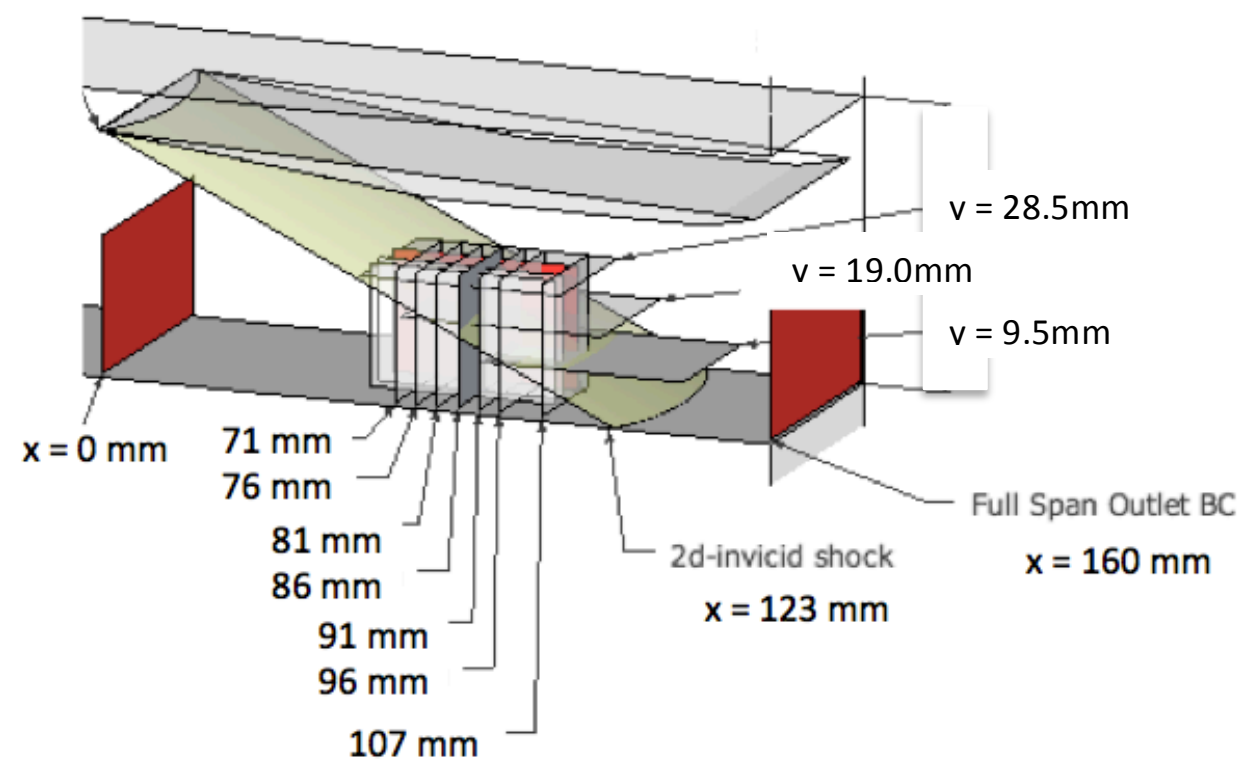

Fig. 2b. Magnified view of the measurement planes seen in Fig. 1a. The plane on the far left is at $x=0$, which is under the leading edge of the shock generator. This $\mathrm{x}=0$ plane is denoted TV2 and is the last condition that the inflow boundary conditions of the SBLI are recorded before the interaction. 9 Transverse planes are denoted by their $\mathrm{x}$ location in $\mathrm{mm}$ in relation to the theoretical 2-D invicid shock reflection point $(123 \mathrm{~mm})$. The vertical height of each streamwise horizontal planes are shown in $\mathrm{mm}$ as well. 


\section{B2. Stereo PIV Diagnostics}

Previously, this "Glass Inlet" wind tunnel has been used to study 2D-SBLI by Lapsa and Dahm [3]. Thus, only a brief outline is presented. Several significant improvements in methodology to align this study with pertinent CFD validation efforts are described. These include pressure, temperature measurements during each run, a correction to the laser triggering and camera timing and a new post-processing method.

Stereoscopic particle image velocimetry (SPIV) produces a single-color cross-correlation PIV measurement. Two interline transfer CCD cameras with 1280x1024 pixel resolution and two Nd:YAG lasers are coordinated by a computer with an onboard programmable timing unit. Each individual particle image is a single-color, double-frame, single-exposure PIV image acquired at an angle 33 degrees to the light-sheet, thus allowing two cameras oriented in a stereo configuration to determine the two in-plane velocity components and the one out-of-plane velocity component over the measurement field-of-view.

To illuminate the seed particles in the field-of-view, a pair of light sheets are created using two frequencydoubled Nd:YAG lasers (one Spectra-Physics Quanta-Ray Pro-250 and one Spectra-Physics GCR-3). The lasers are sequentially triggered to create the double pulse of the $532 \mathrm{~nm}$ sheets, each with total energy of approximately $200 \mathrm{~mJ}$. The flashlamps and Q-switches are triggered by TTL signals at $10 \mathrm{~Hz}$ with a pulse duration of $10 \mathrm{~ns}$. The interframe time between pulses is controlled by a PC-based programmable timing unit (PTU) and the LaVision DaVis 7.2 PIV acquisition software. Both lasers have a $100 \mathrm{~ns}$ intrinsic delay between the rising edge of the trigger pulse and the actual peak in laser energy. This additional delay is significant for the flow conditions used in the present study, and causes the actual interframe time (determined by the peak-to-peak in laser energy) to differ from the target interframe time set by the trigger pulses. Thus the actual delay time between laser pulses is measured before each run with a ThorLabs DET10A/M high-speed photodetector connected to a LeCroy WaveRunner $6030350 \mathrm{MHz}$ oscilloscope. This actual delay time is input to the DaVis software. To do so, the software is told to use its normal internal delay time plus an additional delay that is just enough to cause the total delay in the software to equal the correct (measured) delay time.

The proper laser sheet thickness was set by the following requirement. The laser sheet thickness must be sufficiently large in comparison to the displacement of the particles in the direction perpendicular to the plane. Otherwise there is excessive loss of data if particles imaged in Frame 1 that do not appear in Frame 2. Thus, a thicker sheet is required for the accuracy of the streamwise velocity in the transverse planes, where the high speed $(600 \mathrm{~m} / \mathrm{s})$ streamwise flow is normal to the sheet. Camera hardware limits the inter-frame time to a minimum of approximately $500 \mathrm{~ns}$, so the typical out-of-plane particle displacement is approximately $300 \mu \mathrm{m}$. Good PIV practice is to have a sheet thickness of four times the typical particle displacement in order to minimize particle dropout. So the thicknesses of the transverse planes were set to $1.25 \mathrm{~mm}$. A consistent set of optics was utilized for the streamwise planes, and all sheet thicknesses are listed in Table 1.

\begin{tabular}{l|ccccc}
\hline Image Plane & $\Delta l[\mathrm{~mm}]$ & $d t$ & $D V R_{x}=\frac{u_{\max }}{\sigma_{u}}$ & $D V R_{y}=\frac{v_{\max }}{\sigma_{v}}$ & $D V R z=\frac{\Phi_{\max }}{\sigma_{x}}$ \\
Trvs Full Span & $1.25 \pm 0.25$ & $600 \pm 10$ & $\frac{597}{14}=43$ & $\frac{ \pm 80}{7}=11$ & $\frac{ \pm 80}{7}=11$ \\
Transverse & $1.25 \pm 0.25$ & $600 \pm 10$ & $\frac{597}{14}=43$ & $\frac{ \pm 80}{6}=13$ & $\pm \frac{ \pm 0}{6}=13$ \\
Strm Vert & $1.0 \pm 0.25$ & $600 \pm 10$ & $\frac{597}{7}=85$ & $\frac{ \pm 80}{7}=11$ & $\frac{ \pm 80}{16}=6$ \\
Strm Horz & $1.25 \pm 0.25$ & $600 \pm 10$ & $\frac{597}{8}=74$ & $\frac{ \pm 80}{15}=5$ & $\frac{ \pm 80}{8}=10$ \\
\hline
\end{tabular}

Table 1: Light sheet thickness, $\Delta l$ : laser timing, $d t$ : and three components of the dynamic velocity ratio for each measurement plane computed from the maximum particle displacement and the rms pixel uncertainty in each plane. 


\section{B3. PIV Particle Seeding}

To seed the flow in this study an oil aerosol is generated using a TDA-4B portable Laskin nozzle aerosol generator purchased from ATI Techniques, Inc. The generator consists of an array of six Laskin nozzles that create polydispersed sub-micron particles using a Poly-Alpha Olefin (PAO) oil with density of 819 $\mathrm{kg} / \mathrm{m}^{3}$. This oil also was provided by ATI Techniques. The mean particle diameter is specified to be $0.281 \mu \mathrm{m}$. The particle lag time is the time for a particle velocity to decrease to $1 / \mathrm{e}(37 \%)$ of the change in the gas velocity when the gas undergoes a step decrease in velocity, as it does across a shock wave. The particle time constant depends on particle size and particle density, and is computed to be $0.44 \mu \mathrm{s}$. Samimy showed computationally that particles with Stokes number $\left(\mathrm{St}=\tau_{\mathrm{p}} / \tau_{\mathrm{f}}\right)$ of less than 0.25 will adequately track the large scale motions but that for $\mathrm{St}>0.05$ they were not capable of tracking the smallest scales[15]. For a $M=2.75$ free stream, the characteristic flow time scale at the largest boundary layer condition $\left(\tau_{\mathrm{f}}=\delta / \mathrm{U}\right)$ is $17 \mu \mathrm{s}$, and thus the measured particle Stokes number is $0.44 \mu \mathrm{s} / 17 \mu \mathrm{s}=0.025$ is still within the acceptable range to track the large and small scale motion in the boundary layer and SBLI regions of interest. Of course, there will be larger uncertainty in the velocities measured just downstream of the incident and reflected shock waves. However, these waves are strong only at distances considerably above the sonic line, so we assume that this uncertainty does not significantly affect the data in the SBLI interaction regions. Lapsa [3] measured the particle time lag across a shock wave in the Michigan facility. His results are shown in Fig. 3.

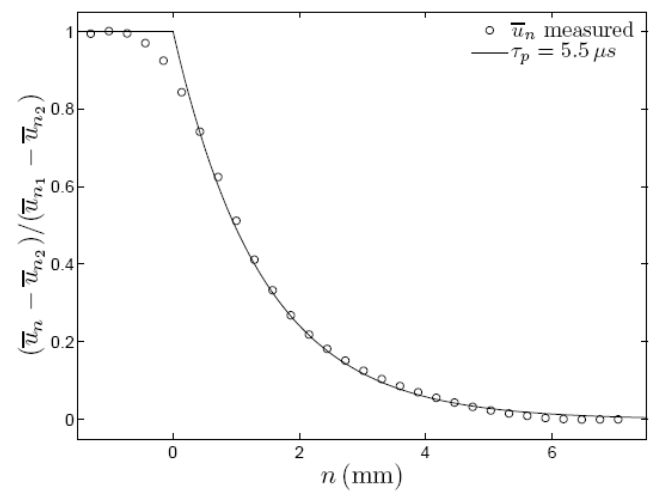

Figure 3. Measured particle response through an oblique shock. The velocity component normal to the shock, $\mathrm{u}_{\mathrm{n}}$, is normalized by the pre-shock $\left(\mathrm{u}_{\mathrm{n} 1}\right)$ and post-shock $\left(\mathrm{u}_{\mathrm{n} 2}\right)$ velocities and shown as a function of the shock-normal direction, $\mathrm{n}$. An exponential fit to the data reveals the particle relaxation time is $5.5 \mu \mathrm{s}$ (Lapsa,A. and Dahm W.J.A., [3]).

The particle images are recorded by two SensiCam PCO interline transfer CCD cameras at $3.33 \mathrm{~Hz}$ (limited by the camera DRAM aquisition). The CCD has a $1024 \times 1280(h \times w)$ pixel array and a physical chip size of $6.8 \times 8.6 \mathrm{~mm}^{2}$. The 12-bit signal depth provides sufficient signal dynamic range, and electronic Peltier cooling ensures low noise. Each camera is equipped with a Sigma 70-300 f/4-5.6 APO macro lens to allow up to $1: 1$ imaging at a minimum focal length of $40.1 \mathrm{~cm}$. For each camera, particle images from the first laser pulse are recorded onto the first frame and then immediately shifted under the submask. The particle images from the second laser pulse are then stored on the second frame.

\section{B4. Prior PIV State of the Art}

PIV techniques have been applied to high-speed flows since the late 90's, and significant progress has been made in the understanding of SBLI in the analysis of this data. However, as can be seen from Table 2 and Table 3, although many such studies have been undertaken, all suffer from a lack of measurement away from the centerline [16-22]. Table 4 presents two recent results on 3D-SBLI, one a tomographic 
PIV data set by Humble and the second, the first PIV measurements made in a sidewall boundary layer by Helmer and Campo.[23-25]

\begin{tabular}{|c|c|c|c|c|}
\hline Author & Beresh 1999 & Hou 2003 & Dupont 2005 & Humble 2006 \\
\hline Mach & $5(750 \mathrm{~m} / \mathrm{s})$ & $2(497 \mathrm{~m} / \mathrm{s})$ & $2.3(550 \mathrm{~m} / \mathrm{s})$ & $2.1(506 \mathrm{~m} / \mathrm{s})$ \\
\hline$\frac{\text { WidthMeasured }}{\text { TunnelWidth }}$ & $\frac{1 \mathrm{~mm}}{152 \mathrm{~mm}}=<1 \%$ & $\frac{1 \mathrm{~mm}}{152 \mathrm{~mm}}=<1 \%$ & $\frac{60 \mathrm{~mm}}{170 \mathrm{~mm}}=35 \%$ & $\frac{4 \mathrm{~mm}}{280 \mathrm{~mm}}=1.5 \%$ \\
\hline & $1.2 \mu s$ & $1 \mu s$ & $1 \mu s-6 \mu s$ & $2 \mu s$ \\
\hline Particle time constant & $54 \mu s$ & $2.6 \mu s$ & No Report & $2.1 \mu \mathrm{s}$ \\
\hline Flow time scale $\delta / U_{\infty}$ & $25 \mu \mathrm{s}$ & $26 \mu s$ & $20 \mu s$ & $38 \mu s$ \\
\hline resolution $\left[\mathrm{mm}^{2}\right]$ & $2 \times 2$ & $0.72 \times 0.72$ & $2.2 \times 1.1$ & $1.9 \times 1.6$ \\
\hline vector spacing & $64 \times 64(50 \%)$ & $32 \times 32$ & No Report & $21 \times 17(75 \%)$ \\
\hline configuration & $\begin{array}{l}\text { boundary } \\
\text { layer }\end{array}$ & $90 \% \mathrm{CR}$ & Full Span OS & $\begin{array}{l}40 \% \text { Span } \\
\text { Oblique }\end{array}$ \\
\hline orientation & Vertical CL & Vertical CL & 6 Horizontal & 5 Vertical \\
\hline number of vector fields & 1500 & 2100 & 500 & 1500 \\
\hline validation method & Pitot & None & LDV & None \\
\hline quantities presented & $\bar{u}, \sqrt{\overline{u^{\prime 2}}}$ & $\bar{u}, \sqrt{\overline{u^{\prime 2}}}$ & $\bar{u}, \sqrt{u_{u^{\prime 2}}}$ & $\begin{array}{l}\bar{u}, \sqrt{\overline{u^{\prime 2}}} \\
\sqrt{\overline{v^{\prime 2}}}, \overline{u^{\prime} v^{\prime}}\end{array}$ \\
\hline
\end{tabular}

Table 2. Previous PIV studies of SBLI 1999-2006

\begin{tabular}{|c|c|c|c|}
\hline Author & Gana... 2006 & Souverein 2008 & Lapsa 2009 \\
\hline Mach & $2(510 \mathrm{~m} / \mathrm{s})$ & $1.69(448 \mathrm{~m} / \mathrm{s})$ & $2.75(600 \mathrm{~m} / \mathrm{s})$ \\
\hline $\begin{array}{l}\text { \% Width Measured } \\
\Delta t\end{array}$ & $\begin{array}{l}\frac{38 m m}{152 m m}=25 \% \\
2 \mu \mathrm{s}\end{array}$ & $\begin{array}{l}\frac{2 \mathrm{~mm}}{280 \mathrm{~mm}}=<1 \% \\
1.5 \mu \mathrm{s}\end{array}$ & $\begin{array}{l}\frac{8 m m}{57 m m}=14 \% \\
0.4 \mu s\end{array}$ \\
\hline Particle time constant & $2.6 \mu s$ & No Report & $5.5 \mu \mathrm{s}$ \\
\hline Flow time scale $\delta / U_{\infty}$ & $26 \mu s$ & $38 \mu s$ & $17 \mu \mathrm{s}$ \\
\hline resolution $\left[\mathrm{mm}^{2}\right]$ & $1.28 \times 1.28$ & $0.43 \times 0.43$ & $0.24 \times 0.24$ \\
\hline vector spacing & $32 \times 32(50 \%)$ & $31 \times 31(75 \%)$ & $32 \times 32(50 \%)$ \\
\hline configuration & Ramp & Oblique & $\begin{array}{ll}60 \% & \text { Span } \\
\text { Oblique } & \end{array}$ \\
\hline orientation & Horizontal & Streamwise & $\begin{array}{l}\text { Transverse and } \\
\text { centerline }\end{array}$ \\
\hline number of vector fields & 750 & $250-4000$ & 1500 \\
\hline validation method & None & High-Res PIV & Repeatability \\
\hline quantities presented & $\bar{u}, \sqrt{u^{\prime 2}}$ & $\bar{u}, \sqrt{\overline{u^{\prime 2}}}$ & $\begin{array}{l}\bar{u}, \bar{v}, \bar{w}, \bar{\omega}_{z}, \bar{\omega}_{x}, \\
\bar{S}_{z y}, \bar{k}, H\end{array}$ \\
\hline
\end{tabular}

Table 3. Previous PIV and SPIV studies 2006-20010 


\begin{tabular}{|c|c|c|}
\hline Author & Humble 2009 & Helmer 2011 \\
\hline Mach & $2.1(510 \mathrm{~m} / \mathrm{s})$ & $2.05(524 \mathrm{~m} / \mathrm{s})$ \\
\hline$\%$ Width Measured & $\frac{14 \mathrm{~mm}}{280 \mathrm{~mm}}=14 \%$ & Inside sidewall BL \\
\hline$\Delta t$ & $2 \mu s$ & $0.8 \mu s$ \\
\hline Particle time constant & $2.6 \mu s$ & $4.4 \mu s$ \\
\hline Flow time scale $\delta / U_{\infty}$ & $40 \mu s$ & $10.3 \mu s$ \\
\hline resolution $\left[\mathrm{mm}^{2}\right]$ & Tomo - $2.1 \times 2.1 \times 2.1$ & $0.135 \times 0.135$ \\
\hline $\begin{array}{l}\text { vector spacing (over- } \\
\text { lap) }\end{array}$ & $42 \times 42 \times 42(75 \%)$ & $16 \times 16$ \\
\hline configuration & Full Span Ramp & $\begin{array}{l}\text { Full Span Ramp / } \\
\text { Oblique Shock }\end{array}$ \\
\hline orientation & Horizontal & 4 Vertical \\
\hline number of vector fields & 750 & $200+$ \\
\hline validation method & None & None \\
\hline quantities presented & inst. $\mathrm{u}, \mathrm{v}, \mathrm{w},\|\omega\|$ & $\bar{u}, \bar{v}, \sqrt{\overline{u^{\prime 2}}} \sqrt{\overline{v^{\prime 2}}}, \overline{u^{\prime} v^{\prime}}$ \\
\hline
\end{tabular}

Table 4. '3D' PIV and Corner Flow Investigations

Since none but the most recent results include sidewall boundary layers, and none provide simultaneous data from across the width of the interaction, the need for measurements which couple together the centerline and sidewall interaction, that include all three components of the velocity in each plane is clearly apparent.

\section{B5. PIV Data Planes}

A total of 16 planes of stereo-PIV data were collected at the locations shown in Fig. 1b. The three streamwise vertical planes are denoted SV1, SV2 and SV3. They are vertical planes that are aligned in the flow direction and thus are parallel to the side wall. The three streamwise horizontal planes are denoted SH1, SH2 and SH3. They are aligned in the flow direction and are parallel to the bottom wall. There are 11 transverse vertical planes. TV0, TV1 and TV2 are where the inflow boundary conditions were measured, and are far upstream of the SBLI. A third transverse vertical plane (TV10) is located far downstream of the SBLI and it is defines the outflow boundary conditions. Stagnation pressure and wall static pressures also were measured at this downstream boundary plane. There are seven transverse vertical planes (TV3 to TV9) that are located in the SBLI region. These data planes are relatively close to each other to better quantify the SBLI region.

The streamwise vertical planes SV1, SV2 and SV3 are sketched in Fig. $2 \mathrm{~b}$ and they have an upstream boundary at the $\mathrm{x}$-locations listed in Table 5 , where $\mathrm{x}=0$ is the leading edge of the shock generator. The dimensions of each plane also are listed. They are located at distances (z) from the sidewall of $17 \mathrm{~mm}, 21$ $\mathrm{mm}$ and $28 \mathrm{~mm}$ respectively.

\begin{tabular}{r|cccccc}
\hline \hline Image Plane & $\mathrm{x}$ & $\mathrm{y}$ & $\mathrm{z}$ & $\Delta x$ & $\Delta y$ & $\Delta z$ \\
\hline SV1 & $70-120$ & $1-27$ & 28 & $0.49 \mathrm{~mm}$ & $0.49 \mathrm{~mm}$ & $1.5 \mathrm{~mm}$ \\
SV2 & $57-100$ & $1-27$ & 21 & $0.49 \mathrm{~mm}$ & $0.49 \mathrm{~mm}$ & $1.5 \mathrm{~mm}$ \\
SV3 & $59-106$ & $1-27$ & 17 & $0.49 \mathrm{~mm}$ & $0.49 \mathrm{~mm}$ & $1.5 \mathrm{~mm}$ \\
\hline \hline
\end{tabular}

Table 5. Dimensions (in $\mathrm{mm}$ ) of the three streamwise vertical planes. $\mathrm{x}=0$ is the leading edge of the shock generator, $\Delta \mathrm{x}$ is the distance between measurement locations on each plane (interrogation box size). 
The streamwise horizontal(SH) planes $\mathrm{SH} 1, \mathrm{SH} 2$ and $\mathrm{SH} 3$ have an upstream boundary that is listed in Table 6 and their dimensions are indicated. They are located at vertical (y-distances) of 9.5, 19 and 28.5 $\mathrm{mm}$ from the bottom wall, respectively.

\begin{tabular}{r|cccccc}
\hline \hline Image Plane & $\mathrm{x}$ & $\mathrm{y}$ & $\mathrm{z}$ & $\Delta x$ & $\Delta y$ & $\Delta z$ \\
\hline SH1 & $90-130$ & 9.5 & $13-44$ & 0.43 & 1.5 & 0.43 \\
SH2 & $78-125$ & 19 & $13-42$ & 0.43 & 1.5 & 0.43 \\
SH3 & $59-106$ & 28.5 & $13-44$ & 0.43 & 1.5 & 0.43 \\
\hline \hline
\end{tabular}

Table 6. Dimensions (in $\mathrm{mm}$ ) of the three streamwise horizontal planes. $\mathrm{y}$ is the distance above the bottom wall. $\Delta \mathrm{x}$ is the distance between measurement locations on each plane (interrogation box size).

The coordinates of the eleven transverse vertical (TV) planes TV0 -TV10 are seen in Table 7. TV0, TV1 and TV2 define the inflow boundary conditions while TV10 defines the downstream boundary conditions available in Part I.

\begin{tabular}{r|rcc}
\hline \hline Image Plane & $\mathrm{x}[\mathrm{mm}]$ & $\mathrm{y}[\mathrm{mm}]$ & $\mathrm{z}[\mathrm{mm}]$ \\
\hline TV0(FS) & -144 & $1-36$ & $1.5-54.5$ \\
TV1(FS) & -72 & $1-36$ & $1.5-54.5$ \\
TV2(FS) & 0 & $1-30$ & $1.5-54.5$ \\
TV3(IR) & 71 & $1-25$ & $2-35$ \\
TV4(IR) & 76 & $1-25$ & $2-35$ \\
TV5(IR) & 81 & $1-25$ & $2-35$ \\
TV6(IR) & 86 & $1-25$ & $2-35$ \\
TV7(IR) & 91 & $1-25$ & $2-35$ \\
TV8(IR) & 96 & $1-25$ & $2-35$ \\
TV9(IR) & 107 & $1-25$ & $2-35$ \\
TV10(FS) & 160 & $1-36$ & $1.5-54.5$ \\
\hline \hline
\end{tabular}

Table 7. Dimensions (in $\mathrm{mm}$ ) of the ten transverse vertical planes. $\mathrm{x}=0$ is the leading edge of the shock generator.

\section{B6. Quality of the Boundary Layer and Repeatability of the Measurements}

The bottom wall of the Michigan facility is a flat plate, as seen in Fig. 1a, in order to insure that the boundary layer is fully-developed and has reached equilibrium. Data in Figure 4 indicate that the velocity profile upstream of the SBLI agrees with classical theory for a fully-developed turbulent boundary layer. These PIV measurements were published previously by Lapsa and Dahm [3].

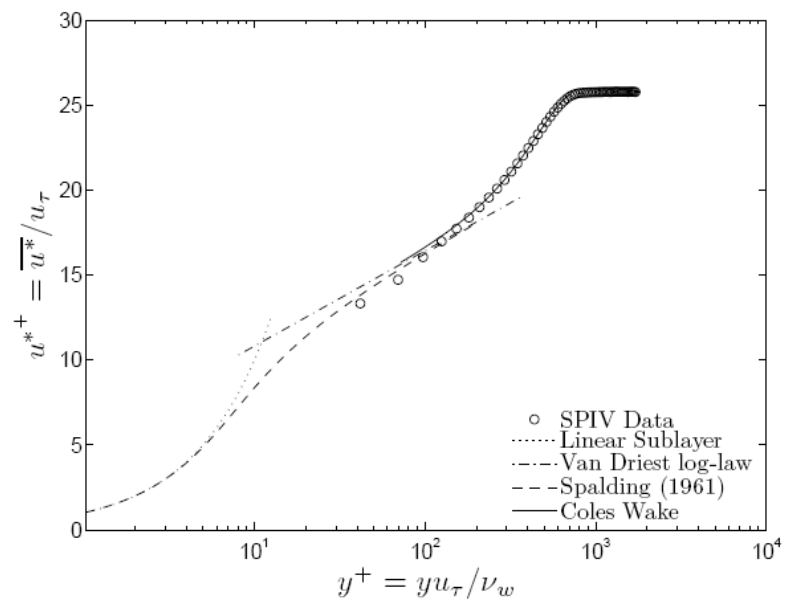


Figure 4. Mean velocity profile measured in the present experiment in the undisturbed boundary layer on the bottom wall, showing that it agrees with the classic theoretical boundary layer structure, using inner variables $\mathrm{u}^{+}$and $\mathrm{y}^{+}$. The plot shows that the entire outer wake-like region is resolved, with the data point nearest the wall extending into the buffer region (Lapsa and Dahm).

The repeatability associated with the present SPIV diagnostics and facility was carefully recorded and a complete data set is available at the Workshop website. Figure 5 indicates that the horizontal and vertical mean velocity profile measurement were repeatable to within 3\% [4].
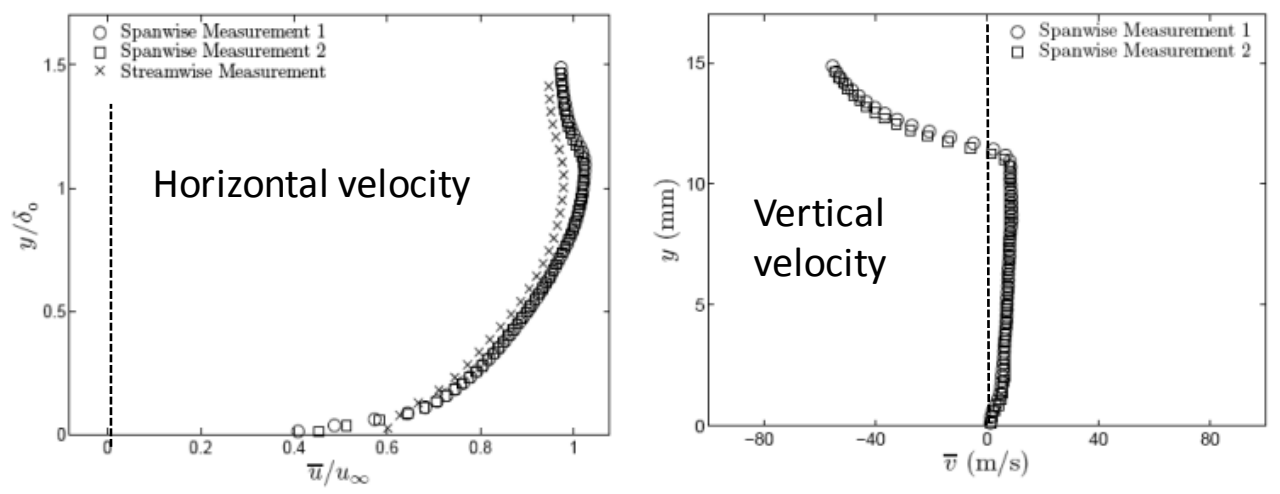

Figure 5. Repeatability of the present Stereo PIV diagnostics and facility, as recorded previously by Lapsa and Dahm [4]. The profiles were recorded on different days.

\section{C.Results}

The results presented in this paper will be limited to a list of the metrics that are being considered and a description of the measurements that were made on the inflow boundary. A complete set of results have been recorded and will be made available at the SBLI Workshop website at a later date.

\section{C.1 Metrics}

One of the goals of the collaborative experimental-computational effort is to decide on the best metrics to use to compare the results from different research teams. In the previous workshop, several metrics were used, but because the current SBLI problem is three-dimensional, additional metrics are needed. The following is a list of useful metrics.

a. Iso-contour curves - of the following quantities, determined in each of the measurement planes (that are defined above):

1. Mean velocity $U, V, W$. Of particular interest is the contour $U=0.9 \mathrm{U}_{\mathrm{oo}}$ that is one definition of the edge of the core flow. Also of interest is the contour $U=220 \mathrm{~m} / \mathrm{s}$ which corresponds to the free stream sonic velocity, and the contour $\mathrm{U}=0$.

2. R.M.S values of fluctuating quantities $u^{\prime}, v^{\prime}, w^{\prime}$ and turbulent kinetic energy k.

3. Reynolds stress velocity correlations: $\overline{u^{\prime} v^{\prime}}, \overline{u^{\prime} w^{\prime}}, \overline{v^{\prime} w^{\prime}}$

4. Normal and shear strain rates $\mathrm{S}_{\mathrm{xx}}, \mathrm{S}_{\mathrm{yy}}, \mathrm{S}_{\mathrm{zz}}, \mathrm{S}_{\mathrm{xy}}, \mathrm{S}_{\mathrm{xz}}, \mathrm{S}_{\mathrm{yz}}$

5. Vorticity components $\omega_{\mathrm{x}}, \omega_{\mathrm{y}}, \omega_{\mathrm{z}}$

6. Vortex-Stream lines, defined by Adrian (see below), which identify vortex structures that are convected with the flow.

\section{b. Profiles}


7. Measured profiles of the above quantities, versus the coordinate that is normal to the wall, have been plotted at selected locations in the interaction region.

\section{c. Additional metrics of importance}

8. Wall skin friction lines, defined as:

$\mathrm{dx} / \tau_{\mathrm{x}}=\mathrm{dz} / \tau_{\mathrm{z}}$ on the bottom wall, and $\mathrm{dx} / \tau_{\mathrm{x}}=\mathrm{dy} / \tau_{\mathrm{y}}$ on the side wall. These skin friction lines can be computed using CFD and can be compared to the measured oil streak lines recorded in this experimental effort.

9. The area of the core flow (where $\mathrm{U}>0.9 \mathrm{U}_{\mathrm{oo}}$ ) is a function of the $\mathrm{x}$-coordinate. This area is a global measure of the effective air flow provided by an inlet. It also is useful metric for determining how close the inlet is to an unstart.

10. Reflected shock locations have been recorded in each of the streamwise vertical planes.

11. The incident boundary layer thickness has been measured and the velocity profile has been shown previously to agree well with a fully-equilibrated Law of the Wall profile.

\section{Vortex-Stream Lines}

Because the flow is three dimensional and highly complex, new visualization methods are needed to properly analyze both the experimental and computational results. PIV developer Ron Adrian, to capture vortex cores convecting in a fast moving freestream, pioneered the vortex-stream line visualization method[26]. These vortex cores are not identifiable when by plotting conventional isolines. Adrian shows that vortex structures are better identified in the x-z plane, for example, by plotting the following isolines:

$$
\frac{d x}{U-U_{A V G}}=\frac{d z}{W-W_{A V G}}
$$

$\mathrm{U}$ and $\mathrm{W}$ are the mean velocity components (ensemble averaged from 1700 images at each (x,z) location in the $\mathrm{x}-\mathrm{z}$ plane), and $\mathrm{U}_{\mathrm{AVG}}$ and $\mathrm{W}_{\mathrm{AVG}}$ are the velocities that are averaged over the entire data plane. Coherent vortices convecting with speed $\left[\mathrm{U}_{\mathrm{AVG}}, \mathrm{W}_{\mathrm{AVG}}\right.$ ] become visible using this method, and shock waves are visible as lines where the vortex-streamlines converge, much like the lines of separation that are identified where the skin friction lines converge.

\section{C2. Freestream Mach Number and Axial Velocity}

The freestream Mach number and axial mean velocity were determined from the ensemble average PIV measurements of $U$ obtained in the undisturbed free stream from regions of intersection of the streamwise and transverse data planes. The data was processed from a small 3 window by 3 window region, and the mean and fluctuating components are reported in Table 8. The corresponding Mach number was determined from the measured stagnation temperature of $295.7 \mathrm{~K}$. Using the isentropic expansion of the nozzle design( $(\mathrm{M}=2.75)$ yields an estimate of free stream temperature of $117 \mathrm{~K}$ and a speed of sound of $217 \mathrm{~m} / \mathrm{s}$. While the methodology for evaluating all the data were the same, the processed data shows some variability. In particular, plane TV1 has both the largest $\mathrm{u}^{\prime}{ }_{\mathrm{rms}}$ and the largest deviation from the mean velocity than the other PIV data in the set. Independent confirmation of this velocity data will be performed with pitot-static probes and will be made available on the website. 


\begin{tabular}{l|llll}
\hline \hline Data Set & {$[\mathrm{x}, \mathrm{y}, \mathrm{z}] \mathrm{mm}$} & $\mathrm{U}(\mathrm{m} / \mathrm{s})$ & $\sigma_{U}$ & $\mathrm{M}$ \\
\hline TV0 & {$[-144,25,17]$} & 598 & 16.1 & 2.756 \\
TV0 & {$[-144,25,28]$} & 602.8 & 16.0 & 2.774 \\
TV0 & {$[-144,25,39]$} & 608 & 15.4 & 2.802 \\
\hline TV1 & {$[-72,25,17]$} & 579 & 23.3 & 2.668 \\
TV1 & {$[-72,25,28]$} & 575 & 24.4 & 2.650 \\
TV1 & {$[-72,25,39]$} & 576 & 26.3 & 2.654 \\
\hline TV2 & {$[0,25,17]$} & 589 & 17.6 & 2.714 \\
TV2 & {$[0,25,28]$} & 591 & 15.5 & 2.723 \\
TV2 & {$[0,25,39]$} & 586 & 13.8 & 2.701 \\
\hline SV1 & {$[73,15,28]$} & 596 & 7.3 & 2.746 \\
TV3 & {$[71,15,28]$} & 603 & 16.5 & 2.779 \\
SV3 & {$[71,15,17]$} & 594 & 8.9 & 2.737 \\
TV3 & {$[71,15,17]$} & 600 & 15.8 & 2.765 \\
\hline \hline & & & Average M & 2.73 \\
& & & std $\sigma_{M}$ & 0.049 \\
\hline \hline
\end{tabular}

Table 8. Free stream Mach number and axial mean velocity determined in thirteen locations. $\sigma_{\mathrm{u}}$ indicates the $u$ component of the rms velocity fluctuation in the measurement.

\section{C3. Inflow Boundary Conditions}

Inflow boundary conditions were measured on three transverse vertical planes (TV0, TV1 and TV2) that were shown in Fig. 1b. Only the measurements on TV2 are presented here. Previously Lapsa and Dahm [3] recorded inflow boundary conditions for this Mach 2.75 facility but they were only interested in the centerline region so their measurements did not extend into the corners or near the sidewall region. Data planes TV0, TV1 and TV2 from near sidewall to sidewall ( $92 \%$ of full span) include the near bottom wall to $48 \%$ of tunnel height. Preliminary results of a validation criteria underdevelopment are presented in Fig 7. Near wall regions that suffer from spurious laser reflection foul the PIV measurement and a robust criterion will be used to discarded these regions from the data set.

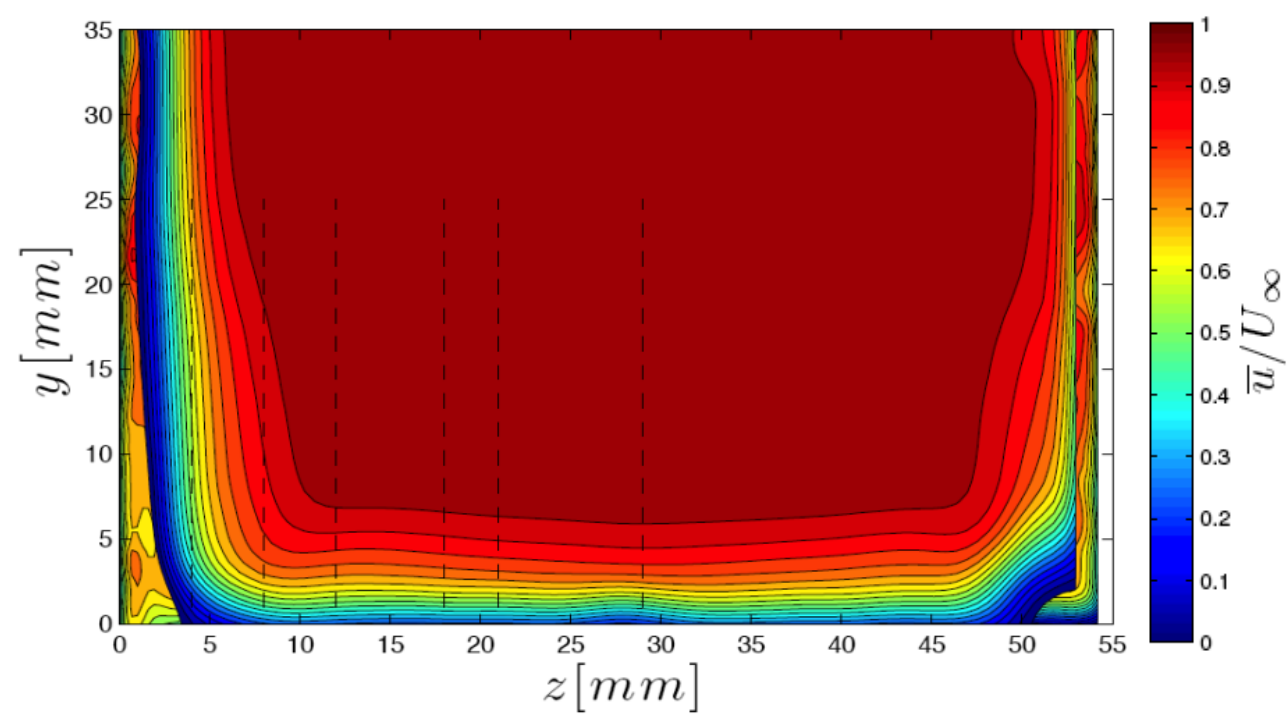

Fig. 6. U-velocity (mean streamwise velocity) on inflow boundary plane TV2. Ensemble average of 1700 images. 


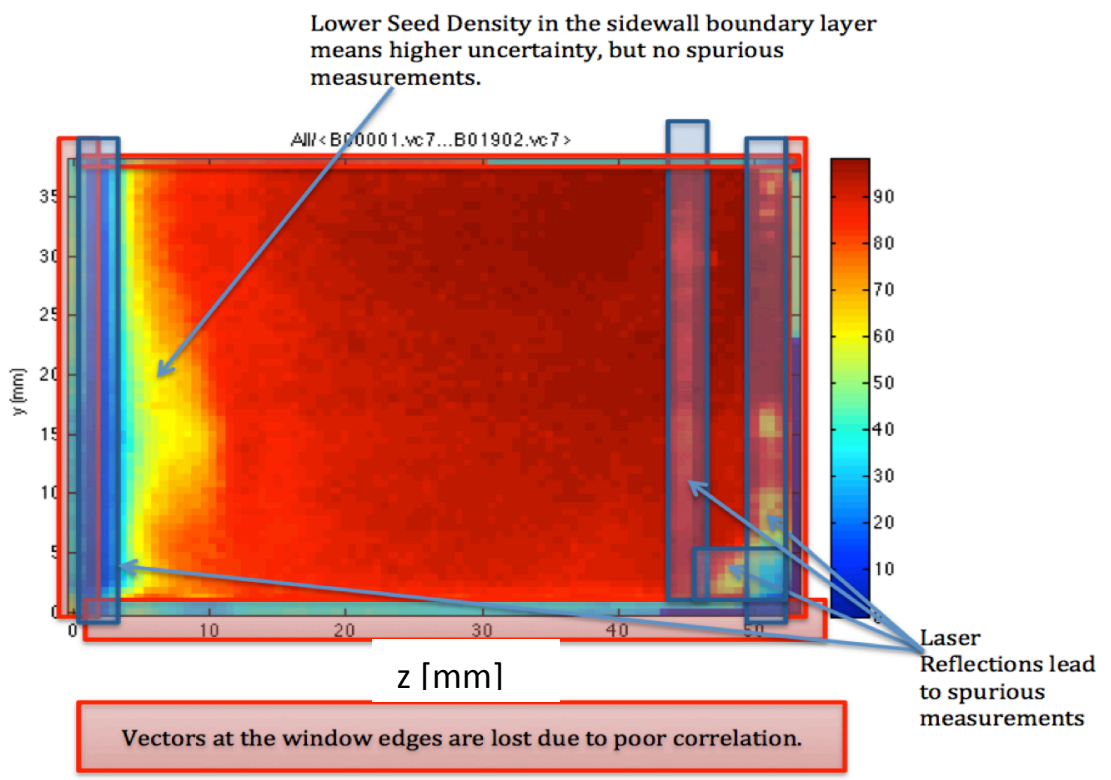

Fig. 7 Spurious data regions indicated using ensemble criterion under development

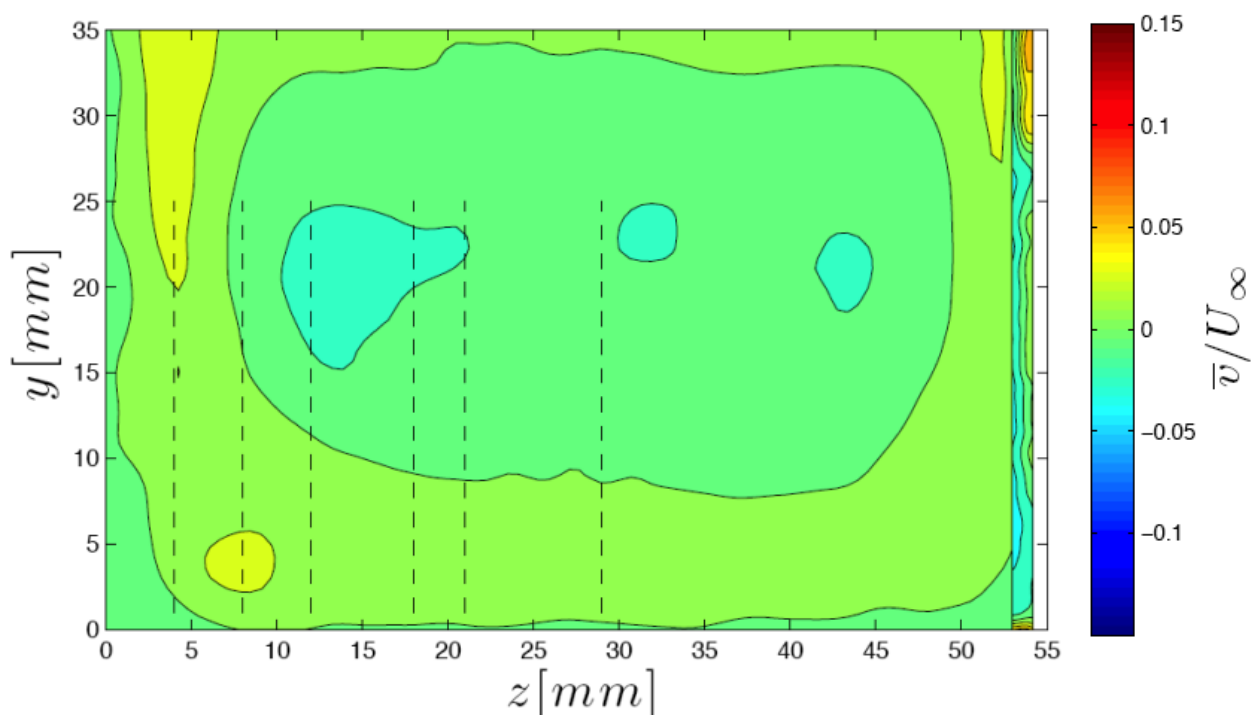

Fig. 8. V-velocity (mean vertical velocity) on inflow boundary plane TV2. Ensemble average of 1700 images. Note that the magnitude of $\mathrm{V} / \mathrm{U}_{\mathrm{oo}}$ is less than $2 \%$. 


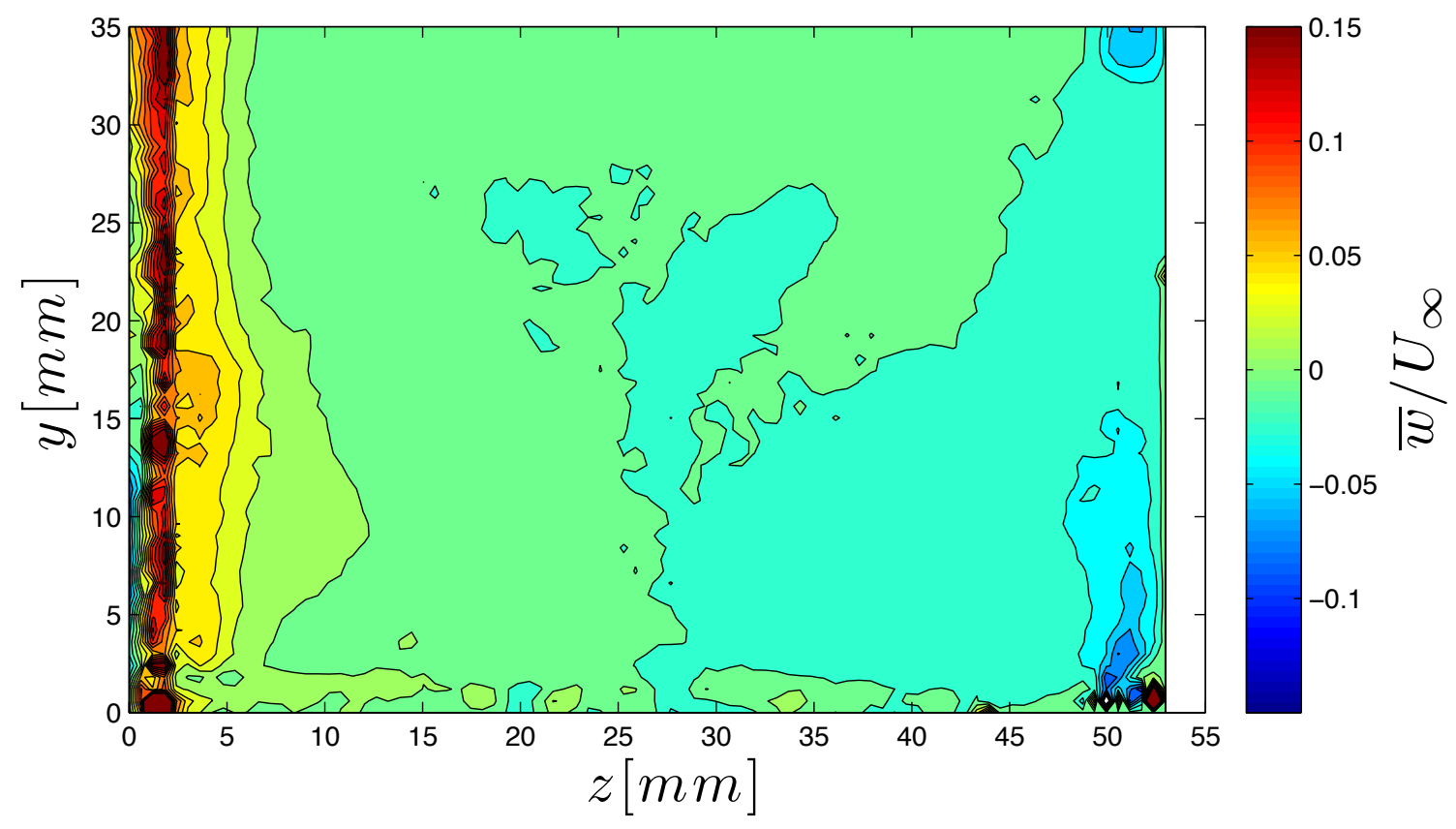

Fig. 9. W-velocity (mean transverse velocity) on inflow boundary plane TV2. Ensemble average of 1700 images. Note that the magnitude of $\mathrm{W} / \mathrm{U}_{\mathrm{oo}}$ is less than $2 \%$. Sidewall data that shows a large deviation is a result of side window reflection. This data will be removed when it is used for comparison.

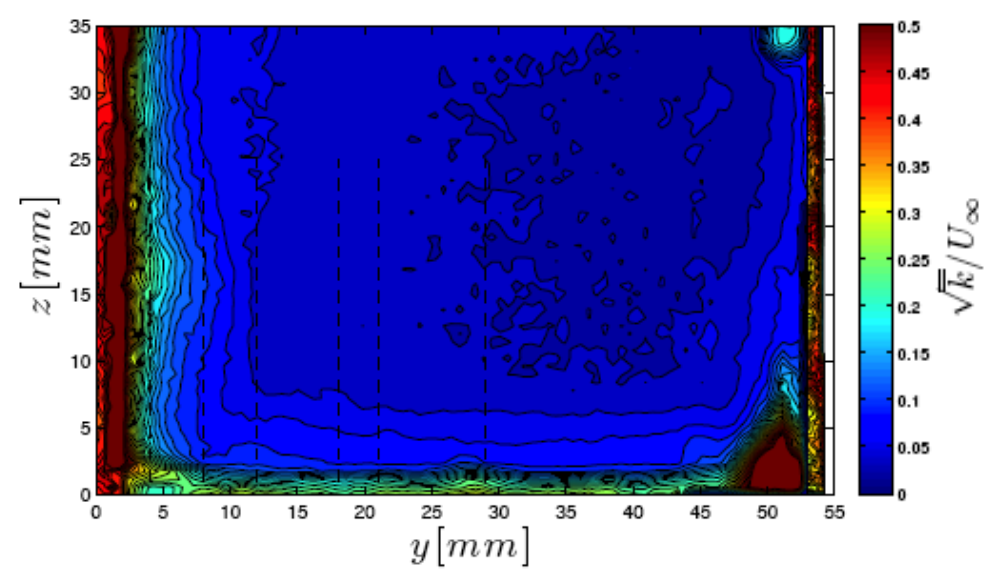

Fig. 10. Turbulence kinetic energy on inflow boundary plane TV2. Ensemble average of 1700 images. Large positive deviations in TKE are due to wall reflections, these spurious data will be removed from the dataset. 

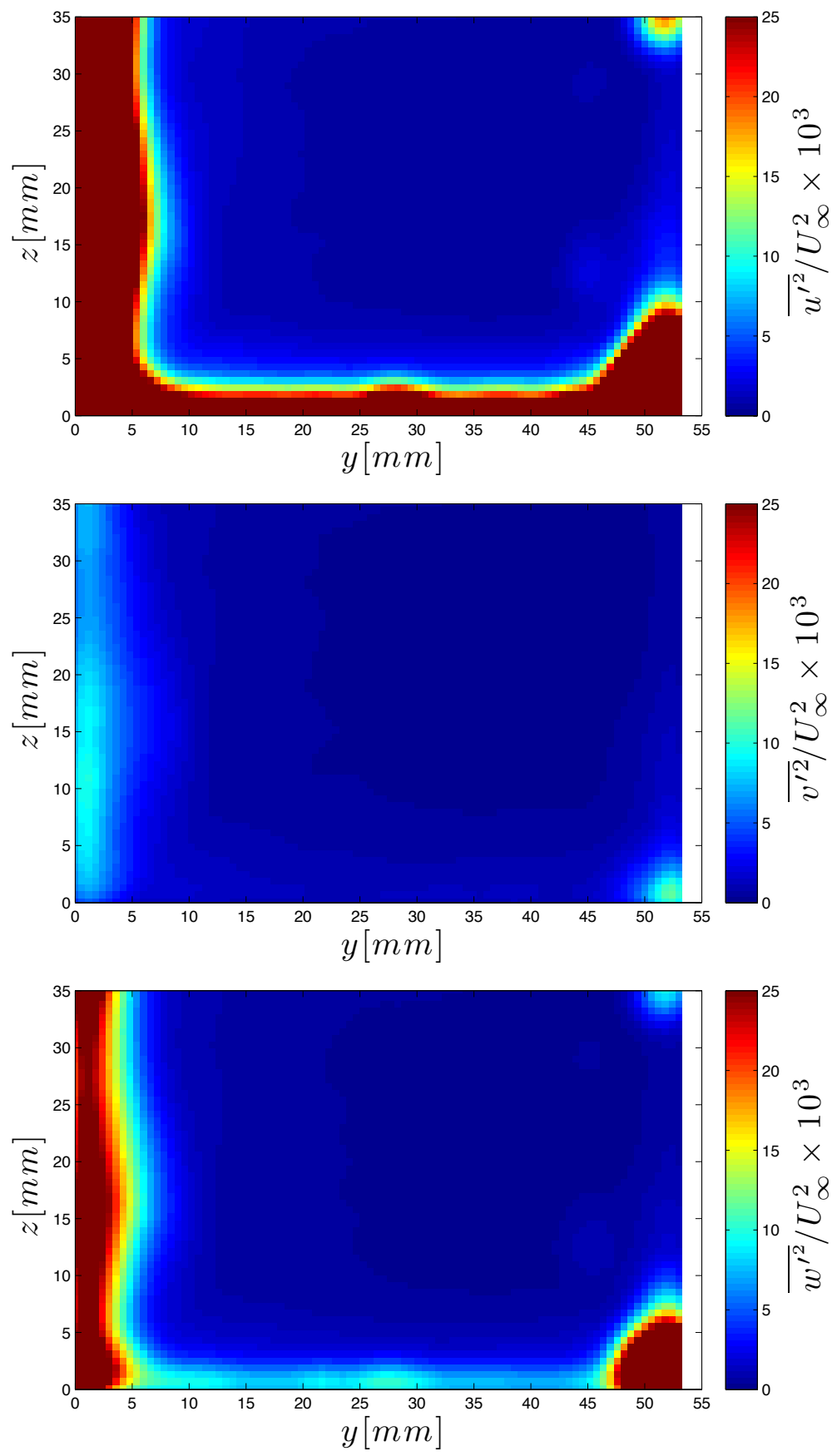

Fig. 11. Mean squared velocity fluctuations on inflow boundary plane TV2. Ensemble average of 1700 images. Large positive deviations in rms are due to reduced signal at the wall. Locations of 'good data' are reported in tables 5-7 above. The lower magnitude of v' in the bottom wall than the sidewall is in good agreement with theory, the very large values of u' and w' in the side wall is due in part to PIV errors in the sidewall data. Figure 14 better compares the magnitudes along the centerline contour. 

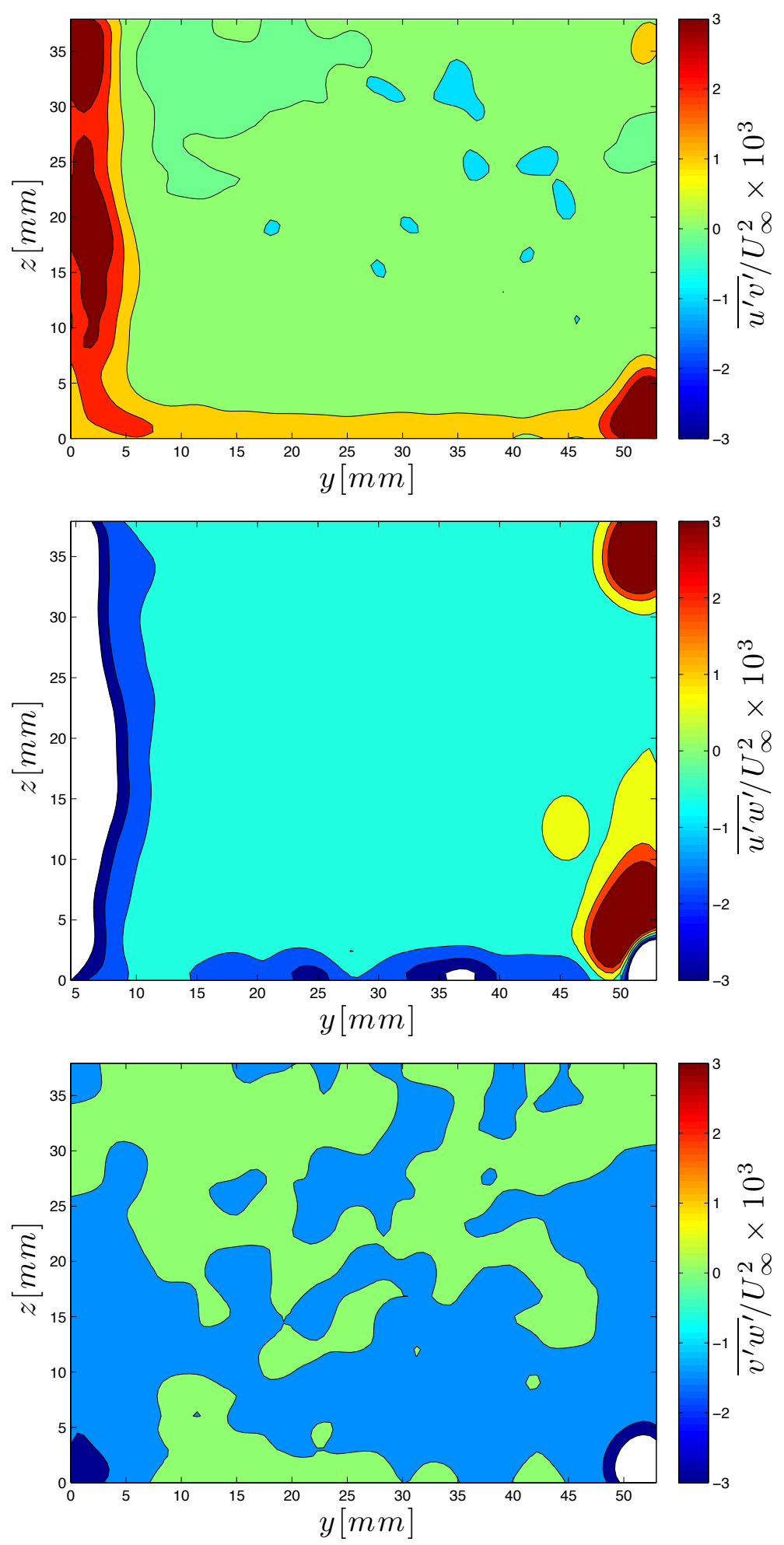

Fig. 12. Reynolds stress velocity correlations on inflow boundary plane TV2. Ensemble average of 1700 images. 


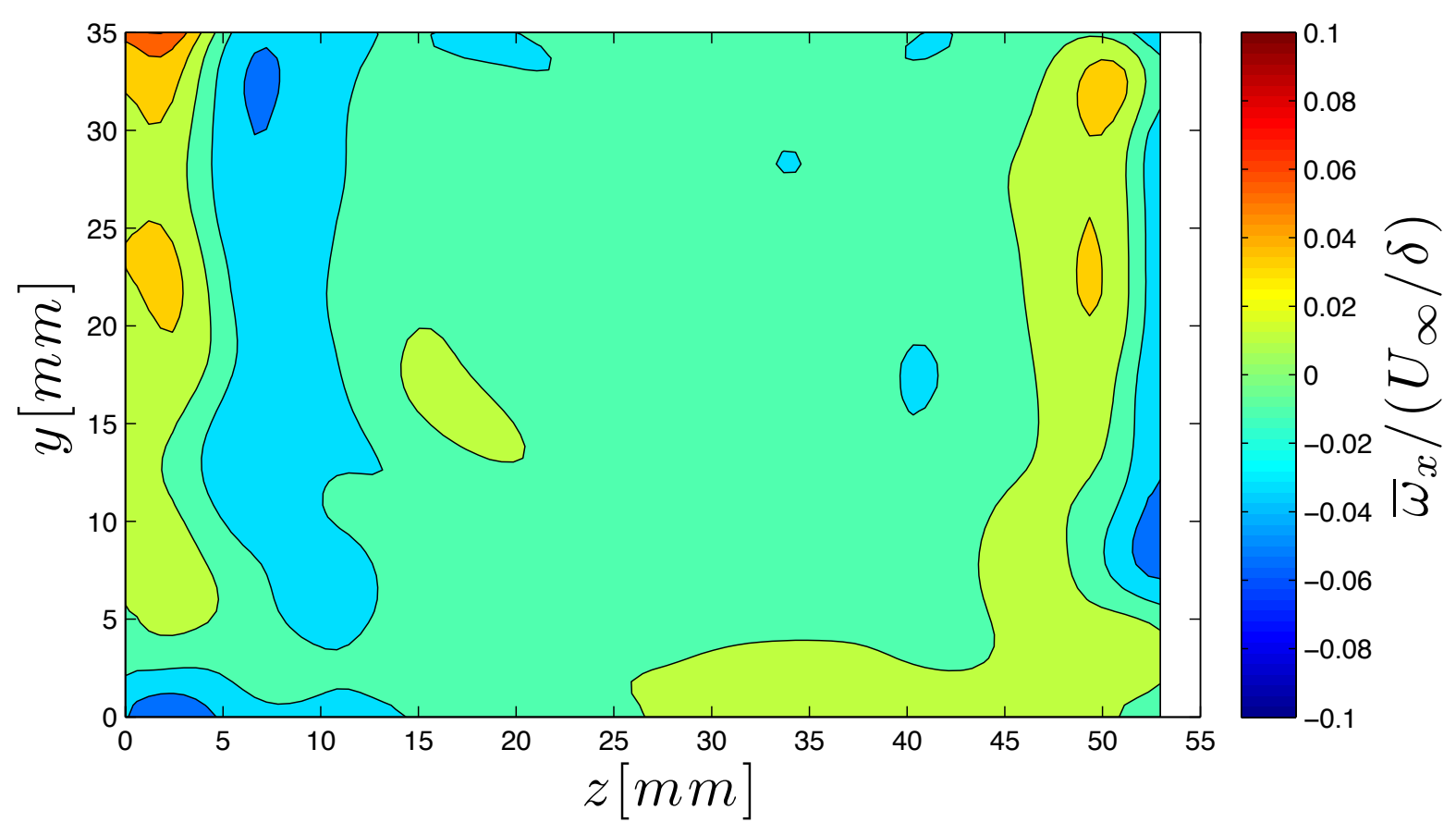

Fig. 13. Mean vorticity $\omega_{\mathrm{x}}$ on inflow boundary plane TV2. Ensemble average of 1700 images.

In the profiles in Figure 14, ensemble averages of the measured quantities on the inflow plane TV2 are presented along a contour at the centerline $(\mathrm{z}=28.5 \mathrm{~mm})$, versus the coordinate that is normal to the wall. We see typical boundary layer behavior in the streamwise direction, and the transverse velocity components are small $(<3 \%)$ at the centerline plane of symmetry. As expected, total turbulence kinetic energy increases sharply very near the wall. The dominant component is the streamwise velocity fluctuation, followed by the transverse component. At the distance to the wall measureable with the PIV in the current experiment there is no appreciable rise in vertical velocity fluctuation. 

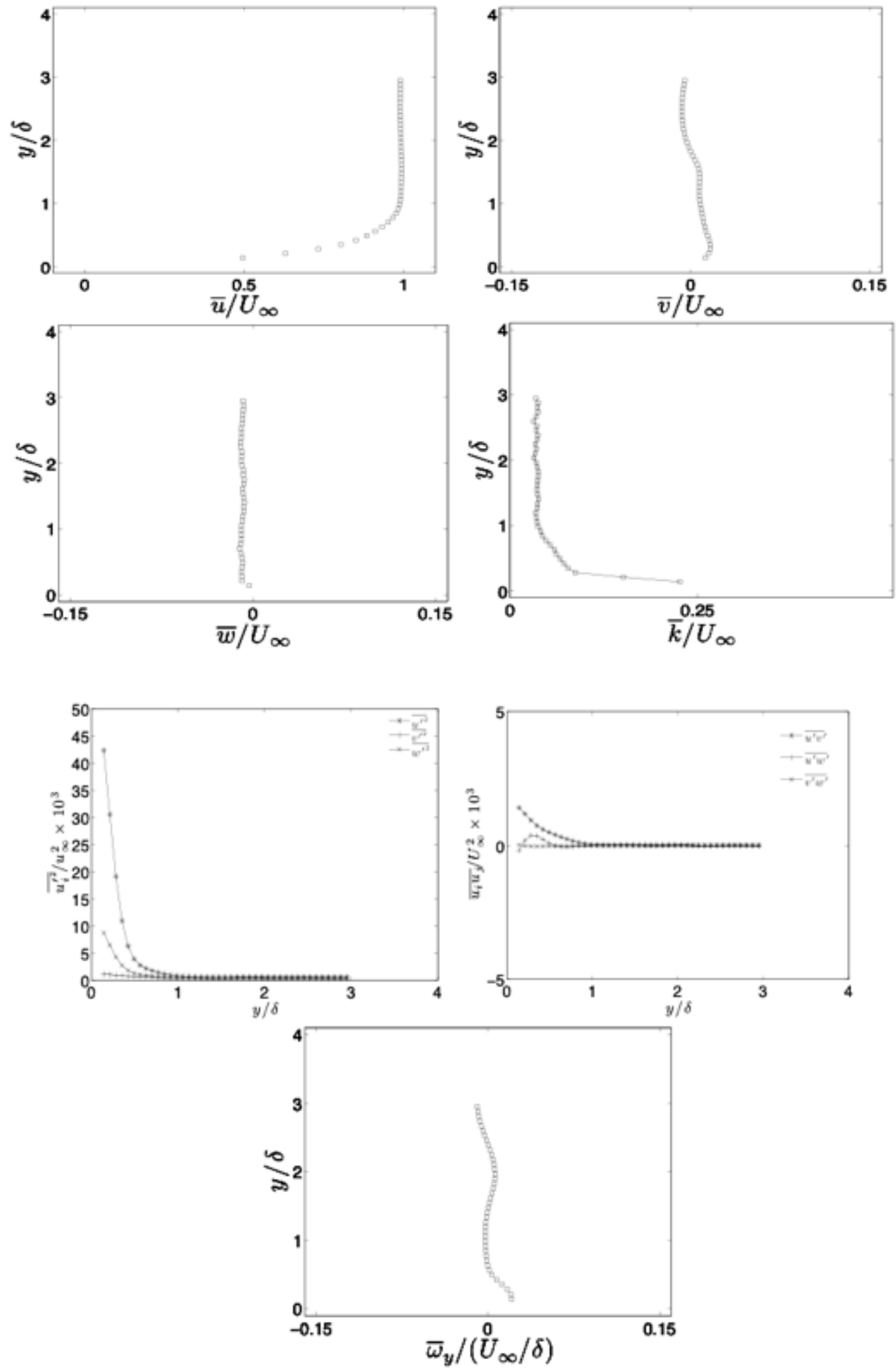

Fig. 14. Some selected profiles of measured quantities on the inflow boundary plane TV2. Ensemble average of 1700 images. 
In each of the PIV data planes 1700 stereo PIV images were recorded. Two methods to average the data were used. Ensemble averaging provided a standard time-average of each of the instantaneous images. Conditional averaging also was used to identify the separation zone in each instantaneous image. Thus, when the separation zone location oscillates in space, the conditional average is a better description of the separation bubble than the ensemble average. Figure 14 lists estimates of the PIV statistical uncertainty, when ensemble averages are determined from 1700 images. The theoretical free stream velocity deviates from the measured value by $0.8 \%$. An uncertainty analysis shows that if 3400 images were recorded rather than 1700 images, the improvement is significantly smaller than other sources of uncertainty. Therefore, efforts to reduce uncertainty should be directed at examining these other sources and not in recording more data. Small uncertainties arise due to day-to-day variation in the room stagnation pressure and temperature (which were recorded and are available one the website), PIV micron sized oil drops lagging behind the flow variations near the shock wave, and possible day-to-day variations in the alignment of the wedge (which was only removed infrequently). Velocity components that were in the PIV laser light sheet plane were measured more accurately than the components that are normal to the plane.

One question that was answered is: "what is the uncertainty associated with selecting 1732 images over which to ensemble average?' Figure 14 lists the PIV statistical uncertainty. The red boxes indicate the size of the $95 \%$ confidence interval. For example, the upper left box indicates that if an ensemble average of 1732 images was recorded, the average value for 1732 images would lie within $0.5 \mathrm{~m} / \mathrm{s}$ of the true statistical mean if only uncertainty associated with the number of images are considered. That is, if more than 1732 images were recorded, there is a $95 \%$ chance that the improvement would be no more than 0.5 $\mathrm{m} / \mathrm{s}$ (and a typical free stream velocity is $600 \mathrm{~m} / \mathrm{s}$ ). It is concluded that 1732 images are sufficient to make the statistical uncertainty significantly smaller than any systematic error. Some sources of systematic error include uncertainty in the time between laser pulses (which was measured with a modern digital scope having a very fast and accurate timing chip), uncertainty in the geometric alignment, the room pressure and temperature (which were measured before each run).

\begin{tabular}{|c||c|}
\hline & Measured \\
\hline$u_{1}[\mathrm{~m} / \mathrm{s}]$ & $602 \pm$ \\
$v_{1}[\mathrm{~m} / \mathrm{s}]$ & $14 \pm$ \\
$u_{2}[\mathrm{~m} / \mathrm{s}]$ & $564 \pm$ \\
$v_{2}[\mathrm{~m} / \mathrm{s}]$ & $-57 \pm$ \\
$\left\|u_{2}\right\|[\mathrm{m} / \mathrm{s}]$ & $566 \pm$ \\
\hline
\end{tabular}

$$
\sigma_{\bar{u}}=\frac{\sigma_{u}}{\sqrt{N}}=\frac{u_{R M S}^{\prime}}{\sqrt{N}}
$$

Free stream: $u_{R M S}^{\prime} \approx 20 \mathrm{~m} / \mathrm{s}$

$$
\begin{gathered}
\bar{u}_{95 \% \mathrm{c}}=\bar{u} \pm 0.5 \mathrm{~m} / \mathrm{s} \\
\text { 3DI-SBLI: } u_{\text {RMS }}^{\prime} \approx 100 \mathrm{~m} / \mathrm{s}
\end{gathered}
$$$$
\bar{u}_{95 \%}=\bar{u} \pm 2.5 \mathrm{~m} / \mathrm{s}
$$

$$
\sigma_{\bar{w}}=\frac{\sigma_{w}}{\sqrt{N}}=\frac{w_{R M S}^{\prime}}{\sqrt{N}}
$$

Free stream: $v_{R M S}^{\prime} \approx 12 \mathrm{~m} / \mathrm{s}$

$$
\begin{aligned}
& \bar{v}_{95 \%}=\bar{v} \pm 0.25 \mathrm{~m} / \mathrm{s} \\
& \text { 3DI-SBLI: } v_{R M S}^{\prime} \approx 75 \mathrm{~m} / \mathrm{s}
\end{aligned}
$$

Free stream: $w_{R M S}^{\prime} \approx 12 \mathrm{~m} / \mathrm{s}$

$$
\bar{w}_{95 \%}=\bar{w} \pm 0.25 \mathrm{~m} / \mathrm{s}
$$$$
\text { 3DI-SBLI: } w_{R M S}^{\prime} \approx 75 \mathrm{~m} / \mathrm{s}
$$

$$
\bar{v}_{95 \%}=\bar{v} \pm 1.9 \mathrm{~m} / \mathrm{s}
$$

$$
\bar{w}_{95 \%}=\bar{w} \pm 1.9 \mathrm{~m} / \mathrm{s}
$$

Figure 15. PIV statistical uncertainty associated with ensemble averaging $\mathrm{N}=1732$ images. The red boxes indicate the size of the $95 \%$ confidence interval. For example, the upper left box indicates that if an ensemble average of 1700 images was recorded, the value for 1700 images would lie within $0.5 \mathrm{~m} / \mathrm{s}$ of the true statistical mean if only uncertainty associated with the number of images are considered. 


\section{D.Discussion of the Physics of 3-D SBLI}

The underlying question that we are trying to answer by comparing CFD to experiment should always be 'With what level of confidence can we assert that two flows the same?' Unlike early experimental efforts requiring 'similarity parameters', a validation effort for CFD attempts to match exactly the experimental conditions. The trouble starts when sources of error confound our attempts to compare the two. In an experiment, the 'exact' fluid equations are being solved, but there is always some non-zero uncertainty in the boundary conditions. Conversely in the CFD, the boundary conditions including the geometry are known precisely, but the fluid equations are being approximated. Thus, in all but the simplest flows, there is bound to be disagreement between measured properties of these two flows, and the disagreement is likely amplified by introducing three-dimensionality.

\section{D1. Three-Dimensional Flows}

In the face of uncertainty introduced by point to point comparisons, larger systems level quantities have been investigated (see the AIAA Drag Prediction workshop... entering its $6^{\text {th }}$ at this very meeting). The idea is that qualitatively the computed and experimental flow are 'the same' if they show the same trends for these integrated quantities, with one simulation being 'better' the closer it can match the experimental data. However, the lack of relative error estimates for each computation and the experiment leaves room for significant improvement. Additionally, there are other three-dimensional metrics which might improve our confidence that the computed flow and the experimental flow are in-fact 'the same' despite differing slightly in detail.

Several previous authors have set out to explain the coupling between three dimensional flow fields and the primitive quantities (as measured by PIV and others or computed in CFD.) The most notable efforts to uncover new 3-dimensional metrics, in the opinion of the current authors, is the efforts of $\mathrm{H}$. Werle, R. Legendre, Dallman, Tobak and Peake; Perry, Chong, and Cantwell [11], [27-29]. These efforts are all related to topological (critical point) methods of fluid analysis. However, these references are mostly conjecture, based upon flow footprints assessed using oil flow and the flow structures are verified only qualitatively using stream trace visualization. Some selected results, though, are remarkable.

The fundamental principle underlying the topological method is that there is a family of three dimensional flow structures that remains stable over a certain range of conditions. If, or when, flow breakdown occurs, the topological structure of the flow also changes, but eventually (in the absence of very strong transient forcing) adopts a new pattern of critical points. The logical extension to be made from the stability of these topologies is that any two flows which share (either experimental or computationally) the same topological features at a given set of nominal conditions could be considered 'the same' in the topological sense. The two flows are 'analogous' since it is only the relative location (not the number or nature) of their topological features which have varied. The lasting worry of these early authors, however, was the lack of uniqueness of a given shear flow profile. Yet, oddly, similar uneasiness (e.g. what is the global flow behavior, or how did it get to be this way) is not given to isolated velocity profiles, so long as they agree with the log-law!

To settle both of these concerns the current validation effort provides both sets; shear stress lines via oil flow and PIV velocity field information. Using the two, a conclusive link between velocity and shear flow can be established. The conclusion drawn from this effort is the nature and distribution of these critical points, is, in fact, critical to correctly assessing the flow features. Going back to the introduction section, we can explain the drawbacks of the first SBLI workshop in this context as being singularly focused on the velocity field without providing a conclusive three-dimensional link to all the boundary conditions. It 
was insufficient in this tunnel, despite a log-law centerline velocity profile, to consider the flow 2D near the centerline.

\section{D2. Dominant Physics}

Incident oblique shocks, compression corners, swept fins and normal shocks generate pressure rises in the boundary layer that result in thickening or separation of the boundary layer [4-14]. Unfortunately, these flows are sufficiently different in structure that each display characteristic scales impeding global parameterization based on two-dimensional analyses and a developed understanding of shock-foot unsteadiness is still a matter of some concern. To assist the computational modeling of this experiment, critical physical aspects of the interactions are illustrated in Fig. 15. The upper figure indicates that vortex structures exist in several places, and they interact. Since RANS computational methods historically have difficulty resolving 'corner flows' accurately, it is imperative that modifications are sought such that each vortex structure that develops is captured in the flow.

The current result also highlights a need for a conceptual shift in our boundary layer flow analysis as we transition from $2 \mathrm{D}$ to $3 \mathrm{D}$ as well as how to interpret results for boundary layers which encounter separation, which will be discussed in terms of SBLI in the following section. To discuss the topology of critical points, we first need to revisit, as others have, the definition of various key terms in the theory. Much of the following is excerpted from Eagle[30], available from DeepBlue thesis retrieval service, starting August 2013.

Begin by considering skin friction. In the simplest 2D case, the skin friction at the wall is a scalar quantity, and is determined locally, for a Newtonian fluid, by the viscosity, and the wall normal gradient of velocity field, $\tau_{\mathrm{w}}=\mu(\mathrm{du} / \mathrm{dy})$. Separation for such $2 \mathrm{D}$ flows is defined to occur where a previously positive shear stress at the wall goes to zero, thus indicating that the local wall normal velocity gradient has also gone to zero. From the steady boundary layer equations this condition occurs when a local pressure gradient has caused the streamwise velocity profile to locally go to zero. For some length downstream of this point the wall shear stress is zero or negative. The flow can remain detached or stalled in the region where the local shear is negative, indicating that the local velocity at this point has reversed. Eventually the flow may reattach, that is, the shear stress will slowly return through zero and become positive again. Such a simple picture is well known in undergraduate fluids courses. However, the extension of this principle to non-periodic, non-two dimensional flows requires significant rethinking. Here enters critical point theory.

In the simplest definition, critical points in the flow are the three dimensional extension of two dimensional separation points. The key difference in three dimensions is that no 'infinite separation line' actually exists (for the three dimensional picture is not a simple extrusion of the 2D case). However, all hope of prediction is not lost. For certain geometries, the locations of many critical points can be predicted a priori given information gleaned from two dimensional experiments. For example, it is possible still to predict when a critical point will occur along a plane of symmetry, due to the somewhat 2 dimensional nature of the flow on this plane. However, when separation occurs in three-dimensional flows there is one key difference from the 2D case. A bifurcation exists in the solution around a single critical point leading to two separate solutions. These solutions depend on the eigenvectors at the critical point, but are more readily described by the secondary flow patterns. There are two types of flow separation, one where the transverse component of velocity converges on the point producing a node and where it diverges from the point producing a saddle. Interestingly these features are indistinguishable when investigating only the plane of symmetry normal to the surface.

This key feature drives us to consider these flows as fundamentally three-dimensional. 
How the shock reflects off the surfaces contribute considerably to vorticity production of the interaction. Where the shock intersects the bottom wall, a lambda shock occurs and straddles a separation zone. The separation zone can be imagined to be a line vortex that is parallel to the bottom wall as shown. Where the shock reflects from the bottom wall, a centerline separation zone occurs. In the corners, two counterrotating vortices exist and define a region of separated flow.

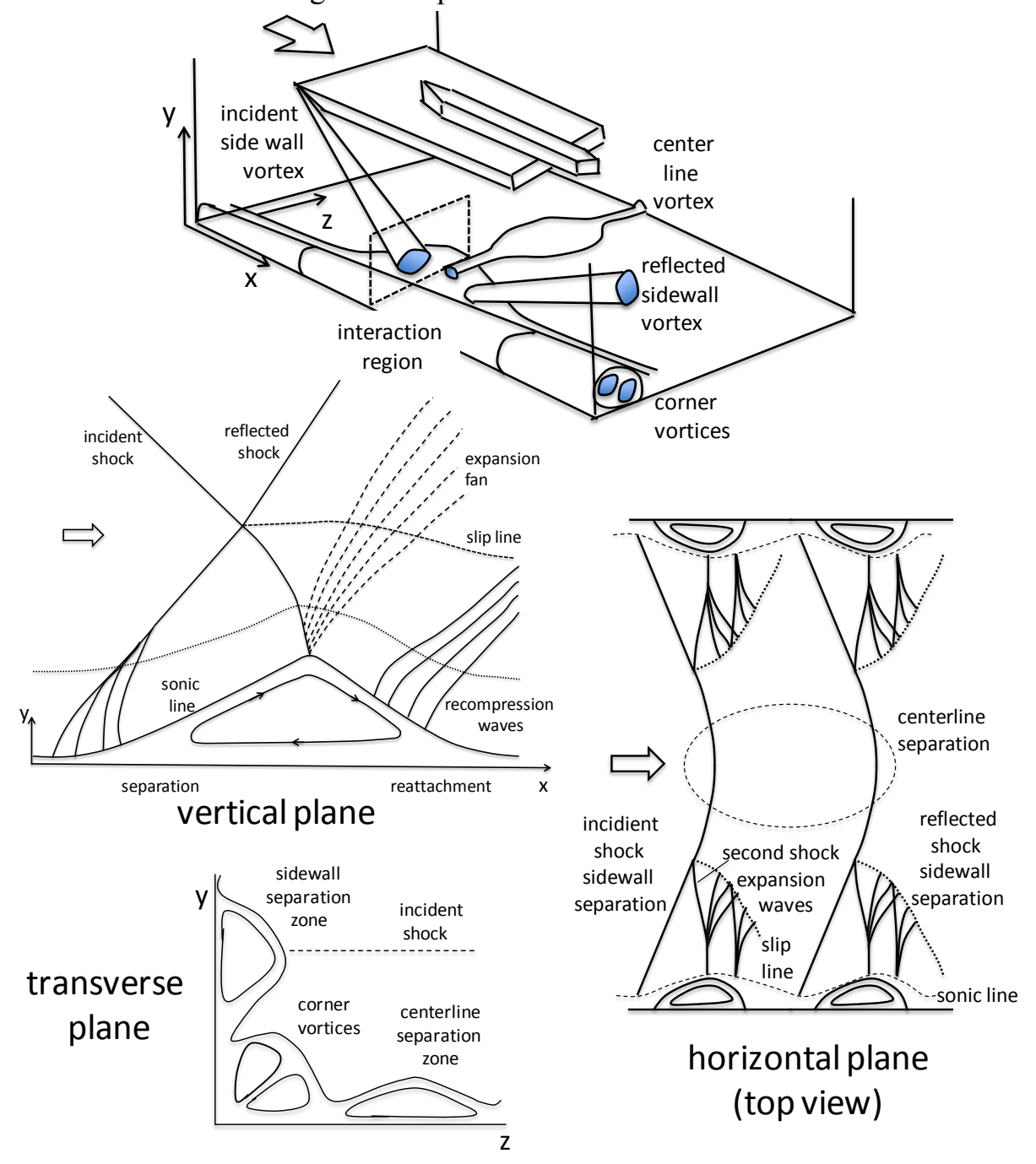

Figure 16. Schematic of the three SBLI unit physics problem that have a strong interaction in a 3-D SBLI in a rectangular inlet.

The mechanism of the corner flow-SBLI appears to be similar to the other SBLI interactions. The primary difference is the strength of the streak line convergence as the bottom wall is approached. The shock from the wedge sweeps along the side wall, converging side wall streak lines and creating down flow toward the bottom wall boundary layer. In the corner, secondary flows created by the adverse pressure gradient due to the original and reflected shocks interact with the turbulent corner flow creating reversed flow and enhancing secondary flow along the bottom wall. The reverse flow moves upstream until it creates a saddle point separation. For small-scale features, the saddle point is very close to the wall, making it difficult to observe. Flow along the bottom wall appears to bend toward the free stream in the region of shock reflection. The stronger the combination of these secondary flows the more severe the corner flow SBLI. 


\section{E. Conclusions}

Improvement in the methodology and careful documentation of experimental data makes this experiment an attractive one for comparison to computation. The computational challenge is to correctly compute all of the underlying physics of a single three-dimensional inlet shockwave boundary layer interaction for the purpose of understanding the prominent interactive features, including aspects of oblique-impinging shocks, crossing shocks, SBLI, and corner flows.

Significant extra care in the experimental design and data gathering phases, including multiple inflow planes, makes this dataset uniquely amenable for CFD validation. In particular, this effort addresses 6 of the 9 areas proposed by Benek[1]. Obviously this includes a full three dimensional interaction, for the assessment of turbulence models in this region(item 1). We also address comprehensive inflow information from 3 data planes(item 2). This data set will provide independent confirmation of the inflow Mach number by pitot, and used flow visualization techniques like oil flow and shadowgraphy combined with wall pressure data in the interaction region (item 3 and 4). Through this process the entire workshop team was invested in the design (item 8 ) and enough information was gathered during each run to provide reliable estimates of experimental error (item 9).

To have a shot at correctly computing this flow, state of the art in CFD techniques will have to capture the fin-plate SBLI, as well as the sidewall-bottom wall effects that propagate through the corner interaction. Strong gradients at the wall and through the shock provide a significant challenge problem for assessing appropriate stress-limiting techniques in CFD such that shocks are well captured, but turbulence intensity is not artificially dissipated. The three-dimensional nature of the separation requires a careful examination of (and explanations for) various behaviors, such as the large-scale vortical structures near the corners, and the change in separation type along the centerline when compared with previous '2D' intuition.

\section{F. Future Work}

The goal of the experimental program has been to keep the total uncertainty in mean quantities below 3\%. To quantify this, estimates of the uncertainty in the present experimental data set will be provided on the Workshop website. There are several sources of uncertainty, including a random uncertainty associated with ensemble averaging over a finite number (1700) of data images, and systematic uncertainties in the experimental setup, measurement techniques and atmospheric condition.

To confirm that this goal has been achieved, pitot pressure and static pressures will be measured to independently confirm the PIV velocity measurements. The resulting free stream velocities will be compared to those in Table 1. That the PIV results already agree to within 3\% is encouraging since the free stream velocity was measured in planes of different orientations on different days. Further comparisons will be attempted in the SBLI interaction region where there are several locations that data overlap among the ten planes to assess the uncertainty using two methods, similar to how the data in first workshop was analyzed by DeBonis et al. [1].

One approach will be based on estimates provided by the experimental team using techniques from PIV uncertainty quantification. The experimental group is developing metrics that utilize the probability distribution function (PDF) of velocities and velocity derivatives at a point. Comparing the velocity pdfs in place of a simple average with an error bar allows us to perform several validation tasks simultaneously. For example, we can visually assess the relative agreement of measurements taken from multiple planes in terms of the average as well as standard deviation, skewness, and kurtosis, and even assess the affect of changing the number of images required to achieve a statistically stationary average. 
Comparison of experiment and computations must also contend with effects of the unsteadiness in the shock foot with the bottom wall and the use of conditional averaging will be employed in these regions to determine intermittency. Finally, some new visualization techniques under development that identify three-dimensional critical points as local invariants of the flow field may hold promise for analyzing and comparing this family of separated flows.

A second independent method to estimate experimental uncertainty will be used that was developed by Aeschliman and Oberkampf, and Oberkampf and Roy [31], [32]. The method is based on statistically analyzing the differences in multiple measurements of the various quantities of interest, e.g., u, v, and w, as well as normal and shear stresses. That is, in the PIV database, measurements are made on different planes. Where these planes intersect there are lines in space where the mean statistical quantities are measured on two different days. In the PIV data set, the streamwise and spanwise data planes have different PIV window sizes, times between laser pulses, and other operating parameters. Several CFD analysts in the first workshop noticed a difference in the PIV data where the two measurement planes intersect. Differences between two independent measurements are not unexpected and examining these differences provides some insights into the fundamental uncertainty in the measurement technique.

\section{Acknowledgements}

The authors would like to take the time to recognize all the SBLI Workshop II organizing committee members: Holger Babinsky (Cambridge, UK), John Benek (AFRL), James Debonis(NASA), Jim Driscoll(University of Michigan), Ethan Eagle (University of Michigan), Charles Hirsch (Numeca, FR), John Luckring(NASA), Bill Oberkampf (WLO Consulting), Paul Orkwis (University of Cincinnati) and Mark Turner (University of Cincinnati).

This work is sponsored by the US Air Force Collaborative Center for Aeronautical Sciences, which is supported by the Air Force Research Laboratory, Air Vehicle Directorate, AFRL/RB and monitored by Dr. John (Jack) Benek, Jon Tinapple, and Lewis Surber.

\section{References}

[1] J. A. Benek, "Lessons Learned from the 2010 AIAA Shock Boundary Layer Interaction Prediction Workshop," AIAA Paper 2010-4825 July 2010.

[2] J. DeBonis et al., "Assessment of Computational Fluid Dynamics and Experimental Data for Shock Boundary-Layer Interaction," AIAA Journal, vol. 50, no. 4, pp. 1-28, 2012.

[3] A. P. Lapsa and W. J. A. Dahm, "Stereo particle image velocimetry of nonequilibrium turbulence relaxation in a supersonic boundary layer," Experiments in Fluids, vol. 50, no. 1, pp. 89-108, Jun. 2010.

[4] P. J. K. Bruce, D. M. F. Burton, N. a. Titchener, and H. Babinsky, "Corner effect and separation in transonic channel flows," Journal of Fluid Mechanics, vol. 679, pp. 247-262, May 2011.

[5] C. W. P. Ford, H. Babinsky, and P. Ford, "Micro-ramp control for oblique shock wave/boundary layer interactions," in 37th AIAA Fluid Dynamics Conference, AIAA 2007-4115, June 2007. 
[6] P. P. Wu and R. B. Miles, "Megahertz Visualization of Compression-Corner Shock Structures," AIAA Journal, vol. 39, no. 8, pp. 1542-1546, Aug. 2001.

[7] A. G. Panaras, "The effect of the structure of swept-shock-wave/turbulent-boundary-layer interactions on turbulence modelling," Journal of Fluid Mechanics, vol. 338, pp. 203-230, May 1997.

[8] F. K. Lu and G. S. Settlest, "Inception length to a fully developed, fin-generated, shock-wave, boundary-layer interaction,” AIAA Journal, vol. 29, no. 5, pp. 758-762, May 1991.

[9] T. Handa and M. Masuda, "Three-Dimensional Normal Shock-Wave/Boundary-Layer Interaction in a Rectangular Duct," AIAA Journal, vol. 43, no. 10, 2005.

[10] P. Doerffer, "An Experimental Investigation of the Math Number Effect upon a Normal Shock Wave -- Turbulent Boundary Layer Interaction on a Curved Wall," Acta Mechanica, vol. 51, 1989.

[11] U. Dallmann, "Topological Structures of Three-Dimensional Vortex Flow Separation," in AIAA 16th Fluid and Plasma Dynamics Conference, AIAA Paper 83-1735, July 1983.

[12] T. Adamson Jr. and A. Messiter, "ANALYSIS OF TWO- DIMENSIONAL INTERACTIONS BETWEEN SHOCK WAVES AND BOUNDARY LAYERS,” Annual Review of Fluid Mechanics, vol. 12, no. 1, pp. 103-138, 1980.

[13] J. Green, "Interactions between shock waves and turbulent boundary layers," Progress in Aerospace Sciences, vol. 11, pp. 235-340, Jan. 1970.

[14] J. D. Schmisseur and D. S. Dolling, "Fluctuating Wall Pressures near Separation in Highly Swept Turbulent Interactions," AIAA Journal, vol. 32, no. 6, pp. 1151-1157, 1994.

[15] M. Samimy and S. K. Lele, "Motion of particles with inertia in a compressible free shear layer," Physics of Fluids A: Fluid Dynamics, vol. 3, no. 8, p. 1915, Aug. 1991.

[16] S. J. Beresh, N. T. Clemens, and D. S. Dolling, "Relationship Between Upstream Turbulent Boundary-Layer Velocity Fluctuations and Separation Shock Unsteadiness," AIAA Journal, vol. 40, no. 12, pp. 2412-2422, Dec. 2002.

[17] Y. X. Hou, N. T. Clemens, and D. S. Dolling, "Wide-Field PIV Study of Shock-Induced Turbulent Boundary Layer Separation," in 41st AIAA Aerospace Sciences Meeting AIAA paper 2003-441, January 2003.

[18] P. Dupont, C. Haddad, and J. F. Debiève, "Space and time organization in a shock-induced separated boundary layer," Journal of Fluid Mechanics, vol. 559, p. 255, Jul. 2006.

[19] R. a. Humble, F. Scarano, and B. W. V. Oudheusden, "Particle image velocimetry measurements of a shock wave/turbulent boundary layer interaction," Experiments in Fluids, vol. 43, no. 2-3, pp. 173-183, Jun. 2007. 
[20] B. Ganapathisubramani, N. T. Clemens, D. S. Dolling, and P. C. Road, "Effects of upstream boundary layer on the unsteadiness of shock-induced separation," Journal of Fluid Mechanics, vol. 585, p. 369, Aug. 2007.

[21] L. J. Souverein, B. W. van Oudheusden, F. Scarano, and P. Dupont, “Application of a dual-plane particle image velocimetry (dual-PIV) technique for the unsteadiness characterization of a shock wave turbulent boundary layer interaction," Measurement Science and Technology, vol. 20, no. 7, Jul. 2009.

[22] A. P. Lapsa, "Experimental Study of Passive Ramps for Control of Shock - Boundary Layer Interactions," University of Michigan, PhD Thesis, 2009.

[23] R. A. Humble, G. E. Elsinga, F. Scarano, and B. W. Van Oudheusden, “Three-dimensional instantaneous structure of a shock wave/turbulent boundary layer interaction," Journal of Fluid Mechanics, vol. 622, no. 1, p. 33, 2009.

[24] D. B. Helmer, "Measurements of a three-dimensional shock-boundary layer interaction," Stanford University, PhD Thesis, 2011.

[25] L. M. Campo, D. B. Helmer, and J. K. Eaton, "Validation Experiment for Shock Boundary Layer Interactions : Sensitivity to Upstream Geometric Perturbations," in 53rd Structures, Structural Dynamics and Materials Conference, AIAA paper 2012-1440, April 2012.

[26] R. J. Adrian, K. T. Christensen, and Z. Liu, "Analysis and interpretation of instantaneous turbulent velocity fields," Experiments in Fluids vol. 29, no. 95, pp275-290 March 2000.

[27] R. Legendre, "Separation de courant l'ecoulement laminarire tridimnesionnel," Rech. Aero, pp. 3$8,1956$.

[28] M. Tobak and D. J. Peake, "Topology of Three-Dimensional Separated Flows," Annual Review of Fluid Mechanics, vol. 14, pp. 61-85, 1982.

[29] M. S. Chong, a. E. Perry, and B. J. Cantwell, "A general classification of three-dimensional flow fields," Physics of Fluids A: Fluid Dynamics, vol. 2, no. 5, p. 765, 1990.

[30] W. E. Eagle, "An Experimental Study of Three-Dimensional Inlet Shock-Boundary Layer Interactions by," 2012.

[31] D. P. Aeschliman, W. L. Oberkampf, and F. G. Blottner, “A Proposed Methodology for Computational Fluid Dynamics Code Verification, Calibration, and Validation," in 16th International Congress on Instrumentation in Aerospace Simulation Facilities, Jul. 1995

[32] W. Oberkampf and C. J. Roy, Verification and Validation in Scientific Computing. Cambridge University Press, 2010. 